\title{
Optimization of energy efficiency of walking bipedal robots by use of elastic couplings in the form of mechanical springs
}

\author{
Fabian Bauer · Ulrich Römer · Alexander Fidlin · Wolfgang Seemann
}

Received: date / Accepted: date

\begin{abstract}
This paper presents a method to optimize the energy efficiency of walking bipedal robots by more than $50 \%$ in a speed range from 0.3 to $2.3 \mathrm{~m} / \mathrm{s}$ using elastic couplings - mechanical springs with movement speed independent parameters. The considered robot consists of a trunk, two stiff legs and two actuators in the hip joints. It is modeled as underactuated system to make use of its natural dynamics and feedback controlled with input-output linearization. A numerical optimization of the joint angle trajectories as well as the elastic couplings is performed to minimize the average energy expenditure over the whole speed range. The elastic couplings increase the swing leg motion's natural frequency thus making smaller steps more efficient which reduce the impact loss at the touchdown of the swing leg. The process of energy turnover is investigated for the robot with and without elastic couplings. Furthermore, the influence of the elastic couplings' topology, its degree of nonlinearity, the mass distribution, the joint friction, the coefficient of static friction and the selected actuator is analyzed. It is shown that the optimization of the robot's motion and elastic coupling towards energy efficiency leads to a slightly slower convergence rate of the controller, yet no loss of stability and a lower sensitivity with respect to disturbances. The optimal elastic coupling discovered by the numerical optimization is a linear torsion spring between the legs.
\end{abstract}

Keywords bipedal robot - dynamic walking - nonlinear feedback control · optimization · energy efficiency · elastic coupling $\cdot$ mechanical spring

F. Bauer · U. Römer $(\bowtie) \cdot$ A. Fidlin · W. Seemann Institute of Engineering Mechanics, Karlsruhe Institute of Technology, Kaiserstraße 10, 76131 Karlsruhe, Germany

Tel.: +49-721-60846823

Fax: +49-721-60846070

E-mail: ulrich.roemer@kit.edu

\section{Introduction}

There are many and varied application scenarios of bipedal robots. The most impressive ones are the humanoid as surrogate of workers in disaster response saving human lives and the exoskeleton as enhancement of disabled people improving human lives. These applications share inherently high mobility requirements, which prohibit an external power supply and therefore directly demand for high energy efficiency. The newly developed robot ATLAS from Boston Dynamics is employed in a disaster response scenario in the Robotics Challenge of the DARPA Program Maximum Mobility and Manipulation (M3). Its $23 \mathrm{~kg}$ lithium-ion-battery provides energy for no more than 20 min of running - the program consistently demands to improve the energy efficiency by a factor of 20 [33]. In order to evaluate the efficiency of locomotion, the dimensionless specific energetic cost of transport $c_{T}=$ energy input $/$ (weight $\times$ distance traveled) is applied. For example, the highly developed humanoid ASIMO from Honda $\left(c_{T}=3.2\right)$ has a 16 times higher specific cost of transport than a human $\left(c_{T}=0.2\right)$ at a low walking speed of $v=0.4 \mathrm{~m} / \mathrm{s}$ [12]. Due to this performance gap between artificial bipedal systems and their biological counterpart, energy efficiency can be justifiably stated as one of the major challenges in the development of walking bipedal robots.

There are essentially two reasons for the bad energy efficiency of todays bipedal robots. First the conservative control strategy: the robot is operated close to static equilibrium, fights against gravitation and tries to suppress any natural dynamics. Humans in contrast walk dynamically, they constantly fall over their feet, swinging their legs like pendulums during walking and therefore exploit their natural dynamics instead of struggling against them [30]. Second the design: the robot consists of rigid links interconnected only by actuated joints. Humans in contrast consist of both rigid skeletons and elastic structures serving as elastic boundary 
layer to absorb shocks, as pogo-stick to redirect center of mass motion and as return spring to buffer energy during the redirection of the swing leg motion [3].

Elastic boundary layers acting as shock absorbers are used in walking robots to reduce the load of the robots' mechanical parts, especially the actuator gear boxes $[24,25$, 28]. Because of their high stiffness compared to other elastic structures, they have a minor influence on the overall system dynamics.

Elastic couplings acting as pogo-stick have been used for a long time in hopping and running robots $[2,36]$ and their influence on the dynamics has been analyzed in detail with the model of the spring loaded inverted pendulum $[6,9,21$, 37].

Elastic couplings acting as return springs have first been used solely in passive dynamic running [29,38]. Later an elastic coupling in the form of a hip spring was also used in spatial passive dynamic walking to adjust the step frequency to the frequency of the toddling motion for stabilization [26]. However, this is not a necessary measure [1]. The idea of adjusting the step frequency by a hip spring was also applied to prevent stumbling by shortening the swing phase of planar passive dynamic walking robots with trunk in simulation [41] and experiment [40].

By enhancing the simplest walking model $[14,20]$ with an elastic coupling in form of a hip spring, the preferred speed-step length relationship of humans could be predicted [27]. With the same model it could be shown that at a given target speed the elastic coupling reduces the step length and therewith the impact loss at touchdown of the swing leg [17]. The robot Cornell Ranger used this effect to walk a marathon at one battery charge [7].

Elastic couplings acting as return springs have also been used in more complex walking models with segmented legs. For a passive model comprising of trunk, two thighs and two shanks it could be demonstrated that an elastic coupling between trunk and thigh is necessary for walking with upright trunk [8]. Using an elastic coupling over multiple joints, a trunkless model with impulsive push off force showed an increase in speed at constant energy input [16].

In addition there was the attempt to rebuild the anatomy of the biological example and its elastic couplings in detail. The robot FastRunner was inspired by an ostrich and uses a network of nonlinear elastic couplings with different modes for the swing phase and the stance phase of each leg. This allows for actuation of the six segmented leg by only one hip actuator [13]. In order to approach human locomotion capabilities, the robot BioBiped was designed [35]. However, its elastic couplings have only been investigated for a hopping motion with given joint torque trajectories without any optimization [34].

On the quest for an energy efficient bipedal robot a collision free gait on level ground was found with a planar pas- sive robot model consisting of trunk, two rigid legs and two rotational springs between trunk and legs [22]. However, the gait without energy costs holds only for one specific speed, is unstable and comes along with a heavy oscillation of the trunk. On an incline this model has shown a much more stable gait than a comparable model without trunk [11].

A big step towards an energy efficient and versatile robot was made by enhancing this model with actuation in the hips $[18,31]$. Stiffness and resting length of hip springs were optimized together with the robot's motion. A significant reduction of actuation could be shown for one constant speed. However, both studies have three major drawbacks, which prevent their practical application: (1) the geometrical and inertial parameters of the investigated robots were far away from humanoid robots, (2) the objective function of the optimization - integral of square of actuator torques over one step - has no physical relationship with energy used and does not account for variable step length and (3) the elastic couplings are optimized only for one specific speed whereas a real robot has to be energy efficient over a range of speeds.

In order to enable the practical applicability of elastic couplings in bipedal robots, these shortcomings are addressed. A process is developed to optimize the motion and the elastic couplings simultaneously over a broad range of speeds with respect to energy efficiency [5]. It allows for a thorough investigation of the effects of elastic couplings to develop an energy efficient robot [4].

This paper is organized as follows. Section 2 introduces the investigated robot model. It consists of the mechanics model (2.1), the actuator model (2.2) and the control strategy (2.3). Section 3 describes the process of generating the motion (3.1) as well as selecting the optimal elastic coupling (3.2) of the robot by simultaneous numerical optimization. Section 4 presents the effect of elastic couplings on the investigated robot. The reduction of specific cost of transport by elastic couplings is explained (4.1) and the influence of the elastic couplings' topology, the robot's mass distribution, joint friction, the coefficient of static friction and the selected actuator (4.2) as well as stability and sensitivity of the motion (4.3) is displayed. Section 5 concludes the investigation, the used methods and the resulting insights and gives an outlook on future research aims. Appendix A provides a more detailed discussion of the results from Sec. 4.2.

\section{Robot model}

The model of the investigated robot consists of the mechanics model (2.1), the actuator model (2.2) and the control strategy (2.3). The approach of modeling the robot as underactuated system and control it with input-output linearization was taken from [39]. 


\subsection{Mechanics model}

The robot is modeled as planar rigid body system. It is composed of two rigid kneeless legs which are separately connected to a rigid trunk via two ideal revolute joints in the hip as depicted in Fig. 1(a). The stance leg foot 1 is modeled a priori as ideal revolute joint and the swing leg foot 2 is assumed to move freely. It will be checked a posteriori, if the unilateral contact and stiction conditions are fulfilled. The ground penetration of foot 2 ("scuffing") is not considered, since the planar model without knees cannot lift its foot. The configuration $\left(\mathbf{q}=\left[q_{1}, q_{2}, q_{3}\right]^{T}\right)$ of the robot is described by two joint angles $\left(\mathbf{q}_{J}=\left[q_{1}, q_{2}\right]^{T}\right)$ and one absolute orientation $\left(q_{A}=q_{3}\right)$. Two actuators between trunk and legs (cf. Fig. 3(a)) apply torques $\left(\mathbf{u}=\left[u_{1}, u_{2}\right]^{T}\right)$ along the respective joint angles $\left(\mathbf{q}_{J}\right)$ and control their evolution. The dynamics of the multibody system can be described by

$\mathbf{M}(\mathbf{q}) \ddot{\mathbf{q}}+\mathbf{Q}(\mathbf{q}, \dot{\mathbf{q}})=\mathbf{B u}$

with the mass matrix $\mathbf{M}(\mathbf{q})$, the vector of generalized forces $\mathbf{Q}(\mathbf{q}, \dot{\mathbf{q}})$ and the input matrix B. The elastic couplings are introduced in Sec. 2.1.3, their restoring torques are included in the vector of generalized forces.

The later prescription of the joint angle trajectories $\left(\mathbf{q}_{J}\right)$ by the control (cf. Sec. 2.3) can be regarded as introduction of virtual constraints. No torques are applied about the point foot 1 or more precisely about the joint towards ground. Therefore, the evolution of the absolute orientation is subject to the remaining dynamics. The bipedal gait consists of two distinct phases which are distinguished by the number of feet on the ground. Adding this discrete state variable to the continuous state variables converts the system to a hybrid system.

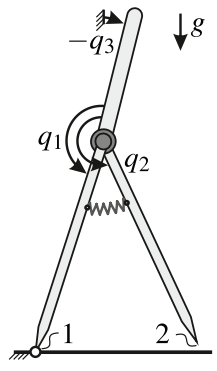

(a)

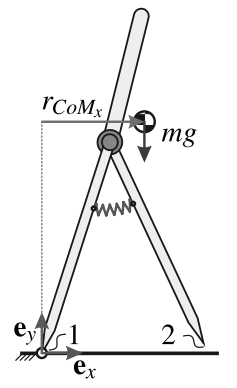

(b)

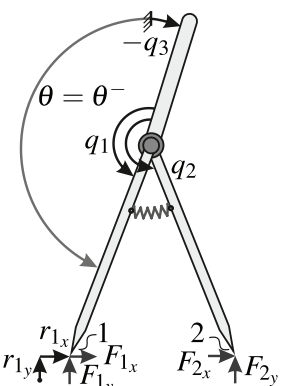

(c)
Fig. 1 Mechanics model of the robot (a) with single support phase as rotation of the total system about foot 1 (b) and double support phase as instantaneous impact of foot 2 (c)

\subsubsection{Single support phase}

During the single support phase solely foot 1 is on the ground. It corresponds to a rotation of the total robot about foot 1 powered by the torque due to the weight $r_{C o M_{x}} m g$ about foot 1 as depicted in Fig. 1(b). The resulting motion can be described by the angular momentum theorem

$\dot{L}_{1}=-r_{C o M_{x}}\left(\mathbf{q}_{J}, q_{A}\right) m g$

with the angular momentum $L_{1}$ of the total system about foot 1 . Since the trajectories of the joint angles $\mathbf{q}_{J}$ are prescribed by the control input $\mathbf{u}$, Eq. (2) corresponds to the remaining dynamics. In order to reflect this situation in the describing equations and to facilitate the control design, Eq. (1) is transformed into the mixed partial feedback linearized normal form. The mass matrix $\mathbf{M}$ does not depend on the absolute angle $q_{A}$ and the torques $\mathbf{u}$ do not directly act on it. Therefore, the equation of motion (1) can be subdivided into $\left[\begin{array}{ll}\mathbf{M}_{J J}\left(\mathbf{q}_{J}\right) & \mathbf{M}_{J A}\left(\mathbf{q}_{J}\right) \\ \mathbf{M}_{A J}\left(\mathbf{q}_{J}\right) & M_{A A}\left(\mathbf{q}_{J}\right)\end{array}\right]\left[\begin{array}{c}\ddot{\mathbf{q}}_{J} \\ \ddot{q}_{A}\end{array}\right]+\left[\begin{array}{l}\mathbf{Q}_{J}(\mathbf{q}, \dot{\mathbf{q}}) \\ Q_{A}(\mathbf{q}, \dot{\mathbf{q}})\end{array}\right]=\left[\begin{array}{c}\mathbf{B}_{J} \\ \mathbf{0}\end{array}\right] \mathbf{u}$.

The angular acceleration of the absolute orientation

$\ddot{q}_{A}=-M_{A A}^{-1}\left(\mathbf{q}_{J}\right)\left(\mathbf{M}_{A J}\left(\mathbf{q}_{J}\right) \ddot{\mathbf{q}}_{J}+Q_{A}(\mathbf{q}, \dot{\mathbf{q}})\right)$

can be calculated with the second row of Eq. (3) and eliminated in the first row. Inverse dynamics determine the joint torques

$$
\begin{aligned}
\mathbf{u} & =\mathbf{B}_{J}^{-1}\left(\overline{\mathbf{M}}\left(\mathbf{q}_{J}\right) \mathbf{v}+\overline{\mathbf{Q}}(\mathbf{q}, \dot{\mathbf{q}})\right), \\
\overline{\mathbf{M}}\left(\mathbf{q}_{J}\right) & =\left(\mathbf{M}_{J J}\left(\mathbf{q}_{J}\right)-\mathbf{M}_{J A}\left(\mathbf{q}_{J}\right) M_{A A}^{-1}\left(\mathbf{q}_{J}\right) \mathbf{M}_{A J}\left(\mathbf{q}_{J}\right)\right), \\
\overline{\mathbf{Q}}(\mathbf{q}, \dot{\mathbf{q}}) & =\left(\mathbf{Q}_{J}(\mathbf{q}, \dot{\mathbf{q}})-\mathbf{M}_{J A}\left(\mathbf{q}_{J}\right) M_{A A}^{-1}\left(\mathbf{q}_{J}\right) \mathbf{Q}_{A}(\mathbf{q}, \dot{\mathbf{q}})\right)
\end{aligned}
$$

as a function of the angular acceleration of the joint angles $\mathbf{v}=\ddot{\mathbf{q}}_{J}$, which becomes the new control input. Furthermore, the absolute orientation of the trunk $q_{A}$ is substituted by the absolute orientation of the stance leg (cf. Fig. 1(c))

$\theta=q_{1}+q_{3}=\mathbf{c}_{\theta} \mathbf{q}$.

The canonical change of coordinates

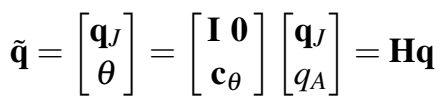

transforms the mass matrix to

$\tilde{\mathbf{M}}\left(\mathbf{q}_{J}\right)=\left(\mathbf{H}^{-1}\right)^{T} \mathbf{M}\left(\mathbf{q}_{J}\right) \mathbf{H}^{-1}$.

The total angular momentum with respect to foot 1

$L_{1}=\left[\tilde{\mathbf{M}}_{A J}\left(\mathbf{q}_{J}\right) \tilde{M}_{A A}\left(\mathbf{q}_{J}\right)\right]\left[\begin{array}{c}\dot{\mathbf{q}}_{J} \\ \dot{\theta}\end{array}\right]$

is the generalized momentum conjugate to $\theta$ and can be determined directly with the mass matrix $\tilde{\mathbf{M}}$ and the angular velocities $\dot{\tilde{\mathbf{q}}}$. Hence, the system equation for the robot's stance phase in first order form can be displayed as

$$
\begin{aligned}
\dot{\mathbf{x}}=\frac{\mathrm{d}}{\mathrm{d} t}\left[\begin{array}{c}
\mathbf{q}_{J} \\
\theta \\
\dot{\mathbf{q}}_{J} \\
L_{1}
\end{array}\right] & =\left[\begin{array}{c}
\dot{\mathbf{q}}_{J} \\
\tilde{M}_{A A}^{-1}\left(\mathbf{q}_{J}\right) L_{1}-\tilde{\mathbf{M}}_{A J}\left(\mathbf{q}_{J}\right) \dot{\mathbf{q}}_{J} \\
\mathbf{0} \\
-r_{C o M_{x}}\left(\mathbf{q}_{J}, \theta\right) m g
\end{array}\right]+\left[\begin{array}{c}
\mathbf{0} \\
\mathbf{0} \\
\mathbf{I} \\
\mathbf{0}
\end{array}\right] \mathbf{v} \\
& =: \mathbf{f}(\mathbf{x})+\mathbf{g}(\mathbf{x}) \mathbf{v}
\end{aligned}
$$


with $\overline{\mathbf{M}}_{A J}\left(\mathbf{q}_{J}\right)=\tilde{M}_{A A}^{-1}\left(\mathbf{q}_{J}\right) \tilde{\mathbf{M}}_{A J}\left(\mathbf{q}_{J}\right)$.

The ground contact force acting in foot 1 can be derived from the free model in Fig. 1(c) and the introduction of the constraint equation

$\mathbf{r}_{1}=\dot{\mathbf{r}}_{1}=\ddot{\mathbf{r}}_{1}=\mathbf{0}$

The configuration vector $\mathbf{q}_{a}=\left[\mathbf{q}^{T}, \mathbf{r}_{1}^{T}\right]^{T}$ is augmented by the position vector $\mathbf{r}_{1}$ of foot 1 . The equation of motion of the free model is given by

$\mathbf{M}_{a}\left(\mathbf{q}_{a}\right) \ddot{\mathbf{q}}_{a}+\mathbf{Q}_{a}\left(\mathbf{q}_{a}, \dot{\mathbf{q}}_{a}\right)=\mathbf{B}_{a} \mathbf{u}+\mathbf{F}_{1, a}+\mathbf{F}_{2, a}$

with the mass matrix $\mathbf{M}_{a}\left(\mathbf{q}_{a}\right)$, the vector of the generalized forces $\mathbf{Q}_{a}\left(\mathbf{q}_{a}, \dot{\mathbf{q}}_{a}\right)$, the input matrix $\mathbf{B}_{a}$ and the vector of the generalized external forces $\mathbf{F}_{1, a}, \mathbf{F}_{2, a}$ of the augmented system. Since the swing leg's foot does not interact with the ground during the single support phase, $\mathbf{F}_{2, a}=\mathbf{0}$. The generalized external force

$\mathbf{F}_{1, a}=\left(\frac{\partial \mathbf{r}_{1}}{\partial \mathbf{q}_{a}}\right)^{T} \mathbf{F}_{1}=\left[\begin{array}{c}\mathbf{0} \\ \mathbf{F}_{1}\end{array}\right]$

can be expressed as projection of the ground contact force acting on foot 1 with the position vector $\mathbf{r}_{1}$ onto the configuration space. Writing Eq. (14) as

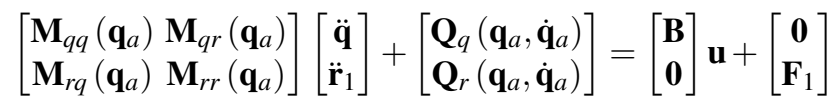

and substituting Eq. (13) into the second line results in

$\mathbf{F}_{1}(\mathbf{q}, \dot{\mathbf{q}}, \mathbf{u})=\mathbf{M}_{r q}(\mathbf{q}) \mathbf{M}^{-1}(\mathbf{q})(\mathbf{B u}-\mathbf{Q}(\mathbf{q}, \dot{\mathbf{q}}))+\mathbf{Q}_{r}(\mathbf{q}, \dot{\mathbf{q}})$,

after elimination of $\ddot{\mathbf{q}}$ with Eq. (1).

\subsubsection{Double support phase}

During the instantaneous double support phase both feet are on the ground. It is modeled as plastic impact of foot 2 onto the ground with simultaneous contact opening in foot 1 (no interaction: $\mathbf{F}_{1, a}=\mathbf{0}$ ), as depicted in Fig. 1(c). After the touchdown, foot 2 is at rest. It neither slips horizontally nor rebounds vertically. The impact duration is assumed to be infinitesimally short, therefore the configuration of the system

$\mathbf{q}_{a}^{+}=\mathbf{q}_{a}^{-}$

does not change over the impact, whereby ${ }^{+}\left({ }^{-}\right)$indicates the respective quantity directly before (after) the impact. The resulting motion can be described by the momentum theorem in integral form

$\mathbf{M}_{a}\left(\mathbf{q}_{a}^{-}\right) \dot{\mathbf{q}}_{a}^{+}-\mathbf{M}_{a}\left(\mathbf{q}_{a}^{-}\right) \dot{\mathbf{q}}_{a}^{-}=\hat{\mathbf{F}}_{2, a}=\lim _{t^{+} \rightarrow t^{-}} \int_{t^{-}}^{t^{+}} \mathbf{F}_{2, a}(t) \mathrm{d} t$ with the generalized impulse

$\hat{\mathbf{F}}_{2, a}=\left(\frac{\partial \mathbf{r}_{2}\left(\mathbf{q}_{a}^{-}\right)}{\partial \mathbf{q}_{a}}\right)^{T} \hat{\mathbf{F}}_{2}$

of the augmented system. The condition for plastic impact

$\dot{\mathbf{r}}_{2}\left(\mathbf{q}_{a}^{+}\right)=\frac{\partial \mathbf{r}_{2}\left(\mathbf{q}_{a}^{-}\right)}{\partial \mathbf{q}_{a}} \dot{\mathbf{q}}_{a}^{+}=\mathbf{0}$

is stated on kinematic level. After combining Eq. (19) - (21) into a system of linear equations they can be solved for the unknowns. The angular velocity of the enhanced system after impact as well as the impulse acting on foot 2

$\left[\begin{array}{c}\dot{\mathbf{q}}_{a}^{+} \\ \hat{\mathbf{F}}_{2}\end{array}\right]=\left[\begin{array}{c}\boldsymbol{\Delta}_{\dot{\mathbf{q}}_{a}}\left(\mathbf{q}^{-}\right) \\ \boldsymbol{\Delta}_{\hat{\mathbf{F}}_{2}}\left(\mathbf{q}^{-}\right)\end{array}\right] \dot{\mathbf{q}}^{-}$

result as linear mapping of the angular velocity before the impact using the identities and abbreviations

$\mathbf{q}_{a}^{-}=\left[\begin{array}{l}\mathbf{I} \\ \mathbf{0}\end{array}\right] \mathbf{q}^{-}, \quad \dot{\mathbf{q}}_{a}^{-}=\left[\begin{array}{l}\mathbf{I} \\ \mathbf{0}\end{array}\right] \dot{\mathbf{q}}^{-}$,

$\boldsymbol{\Delta}_{\hat{\mathbf{F}}_{2}}=-\left.\left(\frac{\partial \mathbf{r}_{2}}{\partial \mathbf{q}_{a}} \mathbf{M}_{a}^{-1}\left(\frac{\partial \mathbf{r}_{2}}{\partial \mathbf{q}_{a}}\right)^{T}\right)^{-1} \frac{\partial \mathbf{r}_{2}}{\partial \mathbf{q}_{a}}\left[\begin{array}{l}\mathbf{I} \\ \mathbf{0}\end{array}\right]\right|_{\mathbf{q}_{a}=\mathbf{q}_{a}^{-}}$,

$\boldsymbol{\Delta}_{\dot{\mathbf{q}}_{a}}=\mathbf{M}_{a}^{-1}\left(\frac{\partial \mathbf{r}_{2}}{\partial \mathbf{q}_{a}}\right)^{T} \boldsymbol{\Delta}_{\hat{\mathbf{F}}_{2}}+\left.\left[\begin{array}{l}\mathbf{I} \\ \mathbf{0}\end{array}\right]\right|_{\mathbf{q}_{a}=\mathbf{q}_{a}^{-}}$.

After the impact the role of the legs is switched, the former stance leg becomes the new swing leg and vice versa. Left and right leg are not distinguished, since only symmetrical gaits are investigated. Using the switching matrix

$\mathbf{R}=\left[\begin{array}{lll}0 & 1 & 0 \\ 1 & 0 & 0 \\ 0 & 0 & 1\end{array}\right]$

the mapping of the multibody state variables over the instance of impact can be stated as

$$
\begin{aligned}
{\left[\begin{array}{c}
\mathbf{q}^{+} \\
\dot{\mathbf{q}}^{+}
\end{array}\right] } & =\left[\begin{array}{c}
\boldsymbol{\Delta}_{\mathbf{q}} \mathbf{q}^{-} \\
\boldsymbol{\Delta}_{\dot{\mathbf{q}}}\left(\mathbf{q}^{-}\right) \dot{\mathbf{q}}^{-}
\end{array}\right], \\
\boldsymbol{\Delta}_{\mathbf{q}} & =\mathbf{R}, \\
\boldsymbol{\Delta}_{\dot{\mathbf{q}}}\left(\mathbf{q}^{-}\right) & =[\mathbf{R} \mathbf{0}] \boldsymbol{\Delta}_{\dot{\mathbf{q}}_{a}}\left(\mathbf{q}^{-}\right) .
\end{aligned}
$$

The angular momentum about foot 2 does not change during impact

$L_{2}^{+}=L_{2}^{-}$.

By changing the reference point of the angular momentum from foot $1^{-}$to $2^{-}$just before the impact and switching the role of the legs from $2^{-}$to $1^{+}$just after the impact the angular momentum of the total system with respect to foot 1 is

$L_{1}^{+}=L_{1}^{-}-r_{2_{x}}\left(\mathbf{q}_{J}^{-}, \theta^{-}\right) m \frac{\mathrm{d} r_{C o M_{y}}\left(\mathbf{q}_{J}^{-}, \theta^{-}\right)}{\mathrm{d} t}$. 
In order to obtain a robust numerical process the impact event - the end of the step and therefore the switching between the single support phase and the double support phase - is defined by the absolute orientation of the stance leg

$\mathscr{S}: \theta=\theta^{-}$

as depicted in Fig. 1(c). To guarantee physically reasonable results of the contact model, the conditions

$r_{2_{y}}\left(\mathbf{q}_{J}^{-}, \theta^{-}\right)=0$

for contact closing of foot 2 at the end of the step and

$\dot{r}_{2_{y}}\left(\mathbf{q}_{J}^{+}, \theta^{+}\right)>0$

for contact opening of foot 1 without interaction at the beginning of the step are introduced.

For both single and double support phase it has to be checked a posteriori, whether the solution fulfills the contact conditions unilaterality

$$
F_{1 y} \leq 0, \quad \hat{F}_{2 y} \leq 0
$$

and stiction ${ }^{1}$

$$
\left|F_{1_{x}}\right| \leq \mu_{0}\left|F_{1_{y}}\right|, \quad \quad\left|\hat{F}_{2_{x}}\right| \leq \mu_{0}\left|\hat{F}_{2_{y}}\right|
$$

\subsubsection{Elastic couplings}

The investigated elastic couplings act with respect to the relative angle $\varphi$ between two bodies. There are two different elementary elastic couplings, the elastic coupling of the legs $\left(l \_l\right)$ and the elastic coupling of the trunk and the legs $\left(t \_l\right)$, the general case is a combination of both as depicted in Fig. 2.

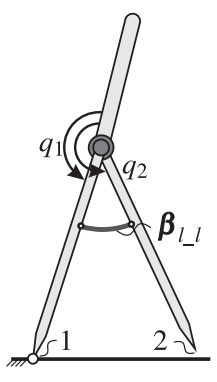

(a)

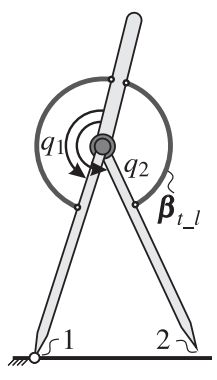

(b)

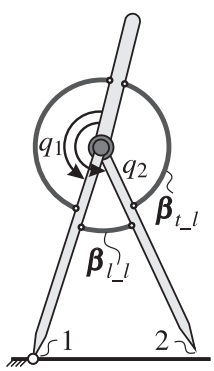

(c)
Fig. 2 Elastic coupling of the legs $\left(l \_l\right)\left(\right.$ a), the trunk and the legs $\left(t \_l\right)$ (b) and the general case as combination of both (c)

\footnotetext{
1 The coefficient of static friction $\mu_{0}$ is assumed to be the same for the continuous motion and the instance of impact. It has been observed that $\mu_{0}$ varies in the impact [10], which is neglected in this paper.
}

The characteristics of each elastic coupling - the torqueangle-relationships - are described by a piecewise power law

$T_{e c}(\varphi)=\left\{\begin{array}{r}-k^{+}\left(\varphi-\varphi_{0}\right)^{v^{+}}, \varphi-\varphi_{0} \geq 0 \\ k^{-}\left(\varphi_{0}-\varphi\right)^{v^{-}}, \varphi-\varphi_{0}<0\end{array}\right.$

as approximation of a general nonlinear relationship. These characteristics can be realized via linear springs with geometric nonlinearities and end stops. The respective joint torque is calculated by

$\mathbf{T}_{J}(\varphi)=T_{e c}(\varphi) \nabla_{\mathbf{q}} \varphi$

with the torque angle relation $\left(T_{e c}\right)$ and the gradient of the relative angle $(\varphi)$ with respect to the configuration variables (q) and is included in the generalized force $\mathbf{Q}(\mathbf{q}, \dot{\mathbf{q}})$ of Eq. (1).

The elastic coupling between the legs acts on the relative angle $\varphi_{l_{-} l}=q_{2}-q_{1}$. It has a vanishing resting angle $\varphi_{l_{-} l_{0}}=0$ and a symmetric torque angle relation $\left(k_{l_{-} l}^{+}=k_{l_{-} l}^{-}=\right.$ $\left.k_{l_{-} l}, v_{l_{-} l}^{+}=v_{l_{-} l}^{-}=v_{l_{-} l}\right)$. Hence, it is defined by two parameters

$\boldsymbol{\beta}_{l_{-} l}=\left[k_{l \_l}, v_{l \_l}\right]$.

The elastic coupling between the trunk and the legs consists of two identical springs which act on the relative angles $\varphi_{t_{-} l_{1}}=q_{1}$ and $\varphi_{t_{-} l_{2}}=q_{2}$. It is defined by five parameters

$\boldsymbol{\beta}_{t_{-} l}=\left[\varphi_{t_{-} l_{0}}, k_{t_{-} l}^{+}, k_{t_{-} l}^{-}, v_{t_{-} l}^{+}, v_{t_{-} l}^{-}\right]$.

The general elastic coupling is a combination of the elementary elastic couplings. It is described by the parameters

$\boldsymbol{\beta}=\left[\boldsymbol{\beta}_{l_{-} l}^{T}, \boldsymbol{\beta}_{t_{-} l}^{T}\right]^{T}$.

These are physical parameters of the robot and therefore movement speed independent. The values of $\boldsymbol{\beta}$ are determined by numerical optimization as described in Sec. 3.2.

\subsection{Actuator model}

Permanent magnet DC motors with transmission by cable and pulleys are selected as actuators as depicted in Fig. 3(b). The realization of the transmission by cable and pulleys combined with a low transmission ratio $i_{T}$ leads to a low frictional resistance. Hence, the actuator is easily backdrivable. Under the assumption of a rigid drive train, the inertia of the actuator is reduced onto the inertia of the attached rigid body and the friction of the actuator is reduced onto viscous joint damping of the actuated joint with the coefficient of joint damping $d_{J}$. Due to the lower time constant in comparison to the mechanical dynamics of the robot, the electrical dynamics are neglected. The actuator remains an ideal torque source within a bounded torque range. 


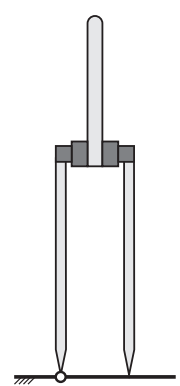

(a)

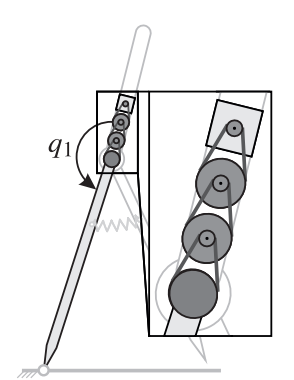

(b)
Fig. 3 Actuator installation circumstances (a) and realization by electric motor with cable and pulley transmission (b)

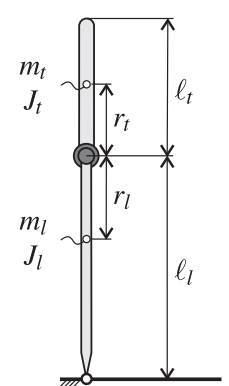

Fig. 4 Geometry and inertia parameters of the rigid body model

In order to quantify the used energy for walking, the electric power input of the actuator

$p_{A}=R_{A} i_{A}^{2}+k_{T} i_{A} \omega_{A}$.

is considered with resistance $R_{A}$, current $i_{A}$ and angular velocity $\omega_{A}$ of the armature and torque constant $k_{T}$. Employing the torque current relationship $T_{A}=k_{T} i_{A}$, the actuator-joint torque relationship $u=i_{T} T_{A}$ and the actuator-joint angular velocity relationship $\omega_{A}=i_{T} \dot{q}$ the electric power

$p_{A}=c_{s t a t} u^{2}+u \dot{q}, \quad c_{s t a t}=\frac{R_{A}}{\left(k_{T} i_{T}\right)^{2}}$

is displayed using merely variables of the rigid body model. The first term in Eq. (43) represents the electric power applied to produce a static torque without performing mechanical work. The second term represents the mechanical power. The coefficient of static electric power $c_{\text {stat }}$ determines how active power is distributed into dissipation power, which is lost as thermal output in the resistance of the armature and usable mechanical power. It is assumed that no electric energy can be recuperated during the operation of the electric motors in generator mode, since the electric circuit is not modeled and its efficiency is unknown. Hence, one actuator's energy consumption during one step

$W_{A}=\int_{0}^{T} \max \left(c_{\text {stat }} u^{2}+u \dot{q}, 0\right) \mathrm{d} t$

is calculated by integration of the positive electric power input over the step duration $T$.

\subsection{Control}

The task of the controller is to realize a predefined motion of the robot by influencing the dynamics of the system. Therefore, the trajectories of the robot's joint angles $\mathbf{q}_{J}$ are controlled. There is a multiplicity of applicable control strategies for nonlinear systems [24, 25, 28,36,42]. The hereinafter presented control approach has not been developed by the authors but is taken from [39].

\subsubsection{Reference trajectory}

The strictly decreasing orientation $\theta$ of the stance leg (cf. Fig. 5) is used as independent variable of the reference trajectories' definition. Compared to time as independent vari-
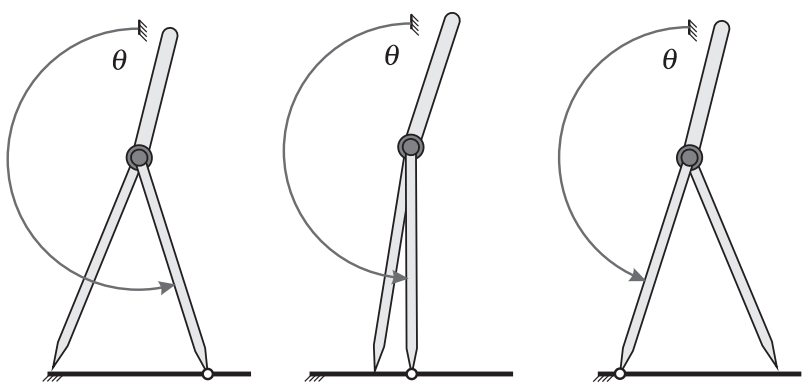

Fig. 5 Strictly decreasing orientation $\theta$ of the stance leg as independent variable for the reference trajectories' definition

able this has the advantage of resulting in an autonomous system. The controller does not enforce the system's phase and the natural dynamics can evolve freely. The reference trajectories are discretized by means of a Bézier curve of degree $n_{\alpha}$

$\mathbf{h}_{r}(\theta)=\sum_{j=0}^{n_{\alpha}} \boldsymbol{\alpha}_{j}\left(\begin{array}{c}n_{\alpha} \\ j\end{array}\right)\left(\frac{\theta-\theta^{+}}{\theta^{-}-\theta^{+}}\right)^{j}\left(1-\frac{\theta-\theta^{+}}{\theta^{-}-\theta^{+}}\right)^{n_{\alpha}-j}$

normalized by the orientation of the stance leg at the beginning $\theta^{+}$and end $\theta^{-}$of a step. The Bézier curve has a smoothening characteristic and contributes to a numerically robust process. It allows for analytical expressions of function value and derivatives at the beginning $\left(\theta^{+}\right)$and the end $\left(\theta^{-}\right)$of a step

$\mathbf{h}_{r}\left(\theta^{+}\right)=\boldsymbol{\alpha}_{0}, \quad \frac{\partial \mathbf{h}_{r}}{\partial \theta}\left(\theta^{+}\right)=\frac{n_{\alpha}\left(\boldsymbol{\alpha}_{1}-\boldsymbol{\alpha}_{0}\right)}{\theta^{-}-\theta^{+}}$,

$\mathbf{h}_{r}\left(\theta^{-}\right)=\boldsymbol{\alpha}_{n_{\alpha}}, \quad \frac{\partial \mathbf{h}_{r}}{\partial \theta}\left(\theta^{-}\right)=\frac{n_{\alpha}\left(\boldsymbol{\alpha}_{n_{\alpha}}-\boldsymbol{\alpha}_{n_{\alpha}-1}\right)}{\theta^{-}-\theta^{+}}$

as function of the Bézier coefficients $\boldsymbol{\alpha}_{i}$. Only periodical gaits with symmetrical steps of left and right foot are investigated. With the impact map Eq. (27) and

$\mathbf{q}=\mathbf{H}^{-1}\left[\begin{array}{c}\mathbf{h}_{r}(\boldsymbol{\theta}) \\ \boldsymbol{\theta}\end{array}\right], \quad \dot{\mathbf{q}}=\mathbf{H}^{-1}\left[\begin{array}{c}\frac{\partial \mathbf{h}_{r}(\boldsymbol{\theta})}{\partial \boldsymbol{\theta}} \\ 1\end{array}\right] \dot{\boldsymbol{\theta}}$

the Bézier parameters at the beginning of the step

$$
\begin{aligned}
{\left[\begin{array}{c}
\boldsymbol{\alpha}_{0} \\
\theta^{+}
\end{array}\right]=} & \mathbf{H} \boldsymbol{\Delta}_{\mathbf{q}} \mathbf{H}^{-1}\left[\begin{array}{c}
\boldsymbol{\alpha}_{n_{\alpha}} \\
\theta^{-}
\end{array}\right], \\
\boldsymbol{\alpha}_{1}= & {\left[\begin{array}{ll}
\mathbf{I} & 0
\end{array}\right] \boldsymbol{\Delta}_{\dot{\mathbf{q}}} \mathbf{H}^{-1}\left[\begin{array}{c}
\boldsymbol{\alpha}_{n_{\alpha}}-\boldsymbol{\alpha}_{n_{\alpha}-1} \\
\frac{n_{\alpha}}{\theta^{-}-\theta^{+}}
\end{array}\right] } \\
& \left(\mathbf{c}_{\theta} \boldsymbol{\Delta}_{\dot{\mathbf{q}}} \mathbf{H}\left[\begin{array}{c}
\frac{n_{\alpha}}{\theta^{-}-\theta^{+}}\left(\boldsymbol{\alpha}_{n_{\alpha}}-\boldsymbol{\alpha}_{n_{\alpha}-1}\right) \\
1
\end{array}\right]\right)^{-1}+\boldsymbol{\alpha}_{0}
\end{aligned}
$$


can be derived from the Bézier parameters at the end of the step $\left(\boldsymbol{\alpha}_{n_{\alpha}}, \boldsymbol{\alpha}_{n_{\alpha}-1}, \theta^{-}\right)$(cf. [39, pp. 141-143]). Hence, the reference trajectories of the joint angles and therefore the motion of the robot can be described by the Bézier parameters

$\boldsymbol{\alpha}=\left[\boldsymbol{\alpha}_{2}^{T}, \ldots, \boldsymbol{\alpha}_{n_{\alpha}}^{T}, \theta^{-}\right]^{T}$

These describe the robot's motion and are therefore movement speed dependent. Their values are determined by numerical optimization as described in Sec. 3.1.

\subsubsection{Hybrid zero dynamics}

The control of the joint angles $\mathbf{q}_{J}$ is realized by input-output linearization. The system's output

$\mathbf{y}=\mathbf{h}(\mathbf{x})=\mathbf{h}_{r}(\boldsymbol{\theta})-\mathbf{q}_{J}$

is defined as the deviation of the joint angles from the reference trajectory. Differentiating the output twice with respect to time using chain rule and Eq. (12)

$$
\begin{aligned}
\dot{\mathbf{y}} & =\frac{\partial \mathbf{h}(\mathbf{x})}{\partial \mathbf{x}} \mathbf{f}(\mathbf{x})=: \mathrm{L}_{\mathbf{f}} \mathbf{h}(\mathbf{x}), \\
\ddot{\mathbf{y}} & =\frac{\partial \mathrm{L}_{\mathbf{f}} \mathbf{h}(\mathbf{x})}{\partial \mathbf{x}} \mathbf{f}(\mathbf{x})+\frac{\partial \mathrm{L}_{\mathbf{f}} \mathbf{h}(\mathbf{x})}{\partial \mathbf{x}} \mathbf{g}(\mathbf{x}) \mathbf{v} \\
& =: \mathrm{L}_{\mathbf{f}}^{2} \mathbf{h}(\mathbf{x})+\mathrm{L}_{\mathbf{g}} \mathrm{L}_{\mathbf{f}} \mathbf{h}(\mathbf{x}) \mathbf{v}
\end{aligned}
$$

relates it to the input $\mathbf{v}=\ddot{\mathbf{q}}_{J}$. A linear PD controller determines the behavior of the output's second derivative

$\ddot{\mathbf{y}}=-\mathbf{K}_{D} \dot{\mathbf{y}}-\mathbf{K}_{P} \mathbf{y}$

and achieves an asymptotically stable characteristic for

$\mathbf{y}=\mathbf{h}\left(\mathbf{q}_{J}, \theta\right)=0$.

In the case of perfect joint angle reference trajectory tracking, the output as well as its time derivatives vanish $(\ddot{\mathbf{y}}=\mathbf{0})$. However, the system has remaining dynamics, the so called zero dynamics, corresponding to the rotation of the total system about foot 1 . The states of these dynamics are not observable by the output and define the zero dynamics manifold

$$
\mathscr{Z}=\{\mathbf{x} \mid \mathbf{y}=\mathbf{0}, \dot{\mathbf{y}}=\mathbf{0}\}
$$

The zero dynamics are asymptotically stable, achieved by the linear controller (cf. Eq. (55)), and invariant with respect to the impact $(\boldsymbol{\Delta}(\mathscr{S} \cap \mathscr{Z}) \subset \mathscr{Z})$ due to the design of the Bézier parameters (cf. Eq. (49) and (50)). The analysis of the multibody system can thus be reduced to the analysis of the hybrid zero dynamics ${ }^{2}$.

\footnotetext{
${ }^{2}$ Zero dynamics of a hybrid system are called hybrid zero dynamics.
}

The single support phase of the hybrid zero dynamics is described by constraining Eq. (52) - (54) to

$\mathbf{q}_{J}=\mathbf{h}_{r}(\theta)$,

$\dot{\mathbf{q}}_{J}=\frac{\partial \mathbf{h}_{r}(\theta)}{\partial \theta} \dot{\theta}$,

$\mathbf{v}_{0}=-\mathrm{L}_{\mathbf{g}} \mathrm{L}_{\mathbf{f}} \mathbf{h}(\mathbf{x})^{-1} \mathrm{~L}_{\mathbf{f}}^{2} \mathbf{h}(\mathbf{x})$

as well as the remaining dynamics from Eq. (12)

$\frac{\mathrm{d}}{\mathrm{d} t}\left[\begin{array}{c}\theta \\ L_{1}\end{array}\right]=\left[\begin{array}{c}\tilde{M}_{A A}^{-1}\left(\mathbf{q}_{J}\right) L_{1}-\overline{\mathbf{M}}_{A J}\left(\mathbf{q}_{J}\right) \dot{\mathbf{q}}_{J} \\ -r_{C o M_{x}}\left(\mathbf{q}_{J}, \theta\right) m g\end{array}\right]=\left[\begin{array}{c}f_{\theta}(\theta) L_{1} \\ f_{L_{1}}(\theta)\end{array}\right]$.

The special structure of Eq. (61) allows for solving the differential equation by quadrature as proposed in [39, pp. 128131]. The time free formulation

$\frac{\mathrm{d} L_{1}}{\mathrm{~d} \theta}=\frac{f_{L_{1}}(\theta)}{f_{\theta}(\theta) L_{1}}$

is solved with separation of variables for the angular momentum $^{3}$

$L_{1}(\theta)=-\sqrt{\left(L_{1}^{+}\right)^{2}+2 V_{0}(\theta)}$.

The term

$V_{0}(\theta)=\int_{\theta^{+}}^{\theta} \frac{f_{L_{1}}\left(\theta^{\prime}\right)}{f_{\theta}\left(\theta^{\prime}\right)} \mathrm{d} \theta^{\prime}$

as well as the time

$t(\theta)=\int_{\theta^{+}}^{\theta} \frac{1}{f_{\theta}\left(\theta^{\prime}\right) L_{1}\left(\theta^{\prime}\right)} \mathrm{d} \theta^{\prime}$

are determined by quadrature as functions of the orientation of the stance leg $\theta$.

The double support phase is described by

$$
\begin{aligned}
L_{1}^{+}= & \delta_{0}\left(\theta^{-}\right) L_{1}^{-} \\
\delta_{0}\left(\theta^{-}\right)= & 1-r_{2_{x}}\left(\mathbf{h}_{r}\left(\theta^{-}\right), \theta^{-}\right) m \\
& \frac{\partial r_{C o M_{y}}\left(\mathbf{h}_{r}\left(\theta^{-}\right), \theta^{-}\right)}{\partial \theta} f_{\theta}\left(\theta^{-}\right)
\end{aligned}
$$

as linear mapping of the angular momentum over the impact event using Eq. (31) for the zero dynamics. The factor $\delta_{0}$ only depends on the orientation of the stance leg at the end of the step and has to be determined only once for a specific set of reference trajectories $(\boldsymbol{\alpha})$.

\footnotetext{
3 Because the definition of $\theta$ is in mathematically positive direction it strictly decreases (cf. Fig. 5) and the angular momentum is negative.
} 
The gait consists of a sequence of single support phases and double support phases. It can be described by the Poincaré map

$P\left(L_{1}^{-}\right)=-\sqrt{\left(\delta_{0}\left(\theta^{-}\right) L_{1}^{-}\right)^{2}+2 V_{0}\left(\theta^{-}\right)}$,

which maps the angular momentum $L_{1}^{-}$at the end of the step $^{4}$ onto the end of the next step, using Eq. (67) in Eq. (64). Its fixed point states the periodic solution of the gait and is explicitly given by

$L_{1}^{-*}=-\sqrt{\frac{2 V_{0}\left(\theta^{-}\right)}{1-\delta_{0}\left(\theta^{-}\right)^{2}}}$.

The stability of the periodic solution $L_{1}^{-*}$ is evaluated by means of the absolute value of the Floquet multiplier, the eigenvalue of the monodromy matrix

$\left|\frac{\partial P\left(L_{1}^{-*}\right)}{\partial L_{1}^{-}}\right|=\delta_{0}\left(\theta^{-}\right)^{2}<1$.

The sensitivity of the stable, periodical solution $L_{1}^{-*}$ is evaluated by means of the relative size

$s_{\mathscr{B}}=\frac{L_{1}^{-\max }-L_{1}^{-\min }}{L_{1}^{-*}}$

of its basin of attraction $\mathscr{B}$ which is bounded on both sides. The upper bound

$L_{1}^{-\max }=-\sqrt{-\frac{2}{\delta_{0}\left(\theta^{-}\right)^{2}} \min _{\theta^{+}<\theta<\theta^{-}} V_{0}(\theta)}$

ensures the forward rotation of the robot, the lower bound

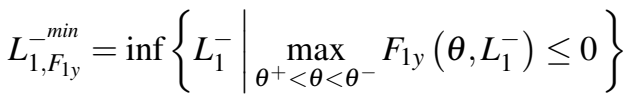

$L_{1}^{-^{m i n}}=\inf \left\{L_{1}^{-}>L_{1, F_{1 y}^{-m i n}}^{-\max }\left|\max _{\theta^{+}<\theta<\theta^{-}}\right| \frac{F_{1 x}\left(\theta, L_{1}^{-}\right)}{F_{1 y}\left(\theta, L_{1}^{-}\right)} \mid \leq \mu_{0}\right\}$

ensures the contact conditions of unilaterality and stiction ${ }^{5}$.

\subsection{Model parameters}

The model parameters used for the investigation of the influence of elastic couplings on the energy efficiency of bipedal robots are displayed in Table 1 and the process parameters in Table 2. In order to analyze effects relevant for practical systems, a $1.80 \mathrm{~m}$ tall and $80 \mathrm{~kg}$ heavy robot is investigated. The

\footnotetext{
4 The touchdown of foot 2 defines the beginning of the step.

5 Substituting Eq. (58) - (67) into Eq. (17) yields the contact force $\mathbf{F}_{1}=\mathbf{F}_{1}\left(\theta, L_{1}^{-}\right)$of the hybrid zero dynamics.
}

Table 1 Model parameters

\begin{tabular}{llrl}
\hline \multicolumn{2}{l}{ Parameter } & Value & Unit \\
\hline$\ell$ & total body length & 1.80 & $\mathrm{~m}$ \\
$m$ & total body mass & 80.0 & $\mathrm{~kg}$ \\
$m_{t}$ & mass trunk & 48.2 & $\mathrm{~kg}$ \\
$m_{l}$ & mass leg & 15.9 & $\mathrm{~kg}$ \\
$\ell_{t}$ & length trunk & 0.533 & $\mathrm{~m}$ \\
$\ell_{l}$ & length leg & 0.892 & $\mathrm{~m}$ \\
$r_{t}$ & center of mass position trunk & 0.289 & $\mathrm{~m}$ \\
$r_{l}$ & center of mass position leg & 0.335 & $\mathrm{~m}$ \\
$J_{t}$ & moment of inertia trunk & 3.09 & $\mathrm{~kg} \mathrm{~m}$ \\
$J_{l}$ & moment of inertia leg & 1.29 & $\mathrm{~kg} \mathrm{~m}{ }^{2}$ \\
$g$ & standard gravity & 9.81 & $\mathrm{~m} / \mathrm{s}^{2}$ \\
$\mu_{0}$ & coefficient of static friction & 0.6 & - \\
$i_{T}$ & transmission ratio & $30: 1$ & - \\
$c_{s t a t}$ & coefficient of static power & $1.81 \mathrm{e}-3$ & $\mathrm{~W} /(\mathrm{Nm})^{2}$ \\
$d_{J}$ & reduced joint damping parameter & 8.00 & $\mathrm{Nms} / \mathrm{rad}$ \\
$n_{\alpha}$ & degree of Bézier curve & 11 & - \\
\hline
\end{tabular}

segmentation and distribution of mass is assumed to be human like. Therefore, the geometrical and inertial parameters (cf. Fig. 4) are determined by regression according to [15]. The rigid bodies' centers of mass, masses and moments of inertia already include the inertia and reduced inertia of the actuators. The coefficient of static friction $\mu_{0}$ is estimated conservatively (cf. Sec. 4.2). The parameters concerning the actuators $\left(i_{T}, c_{\text {stat }}, d_{J}\right)$ are derived from the actuators of the walking robot MABEL $[23,32]$. The determined joint damping parameter $d_{J}$ is merely used for a realistic quantification of the specific cost of transport savings in Sec. 4.2. In the remainder of the paper, the joint damping is neglected. The selected degree of Bézier curve $n_{\alpha}$ is sufficient and does not constrain the optimal motion.

\section{Optimization process}

In this section the generation of motion as well as the selection of elastic couplings by means of numerical optimization is described.

\subsection{Trajectory optimization}

In order to find an energy efficient motion, the trajectories of the joint angles $\mathbf{q}_{J}$ are optimized by determining the Bézier parameters $\boldsymbol{\alpha}$ for a specific movement speed. As objective function

$f_{\min _{\alpha}}(\boldsymbol{\alpha}, \boldsymbol{\beta})=c_{T}=\frac{\sum_{i=1}^{2} \int_{0}^{T} \max \left(c_{s t a t} u_{i}^{2}+u_{i} \dot{q}_{i}, 0\right) \mathrm{d} t}{m g r_{2_{x}}\left(\theta^{-}\right)}$

the dimensionless specific cost of transport is selected, defined as energy input of the actuators (cf. Eq. (44)) divided by weight and step length. 
Table 2 Process parameters

\begin{tabular}{lll}
\hline \multicolumn{2}{c}{ Parameter } & Value \\
\hline$n_{\theta}$ & Number of quadrature sampling points & 401 \\
TolFun & SQP algorithm termination function tolerance & $1 \mathrm{e}-2$ \\
TolCon & SQP algorithm termination constraint tolerance & $1 \mathrm{e}-5$ \\
$n_{\bar{v}}$ & Number of averaging sampling points & 10 \\
TolMesh & PS algorithm termination mesh tolerance & $1 \mathrm{e}-5$ \\
\hline
\end{tabular}

Besides minimizing the objective function, the trajectory of the solution $\boldsymbol{\alpha}^{*}$ of the optimization has to fulfill equality and inequality constraints. The equality constraints $c_{i}(\boldsymbol{\alpha})=$ 0 are given by

$$
\begin{gathered}
\bar{v}-\frac{r_{2_{x}}\left(\theta^{-}\right)}{t\left(\theta^{-}\right)}=0, \\
r_{2_{y}}\left(\theta^{-}\right)=0
\end{gathered}
$$

and consist of the desired average horizontal velocity $\bar{v}$, in this paper referred to as speed, and contact closing at the end of the step (cf. Eq. (33)). The inequality constraints $c_{i}(\boldsymbol{\alpha}) \leq$ 0

$$
\begin{array}{rlrl}
F_{1_{y}} & \leq 0, & \hat{F}_{2_{y}} \leq 0, \\
\left|F_{1_{x}}\right|-\mu_{0}\left|F_{1_{y}}\right| & \leq 0, & \left|\hat{F}_{2_{x}}\right|-\mu_{0}\left|\hat{F}_{2_{y}}\right| \leq 0, \\
-\dot{r}_{2_{y}}\left(\theta^{+}\right) & \leq 0 & &
\end{array}
$$

consist of unilaterality (cf. Eq. (35)), stiction (cf. Eq. (36)) and contact opening without interaction (cf. Eq. (34)). To solve the nonlinear, constrained optimization problem the SQP algorithm is utilized.

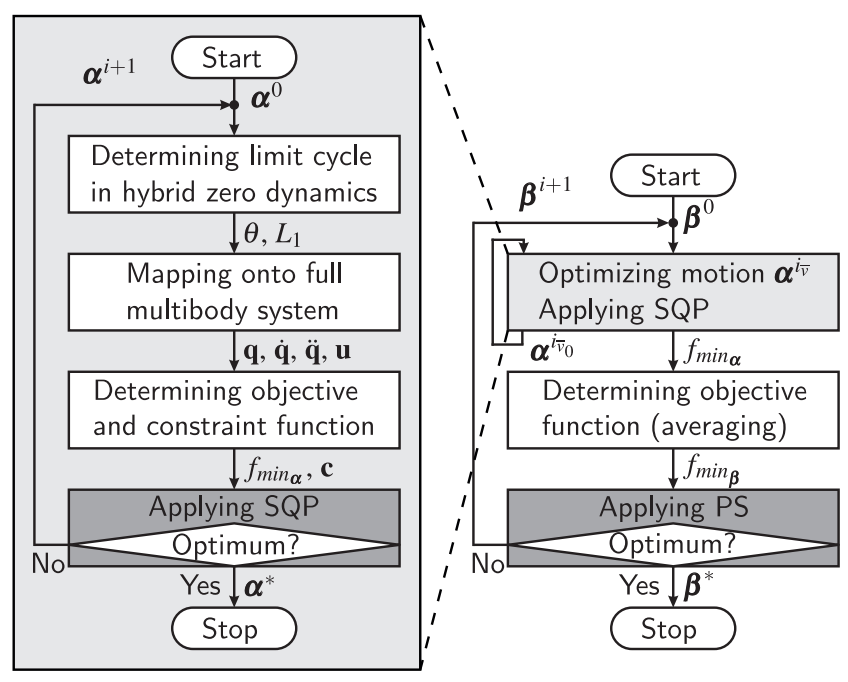

(a)

(b)

Fig. 6 Process of optimizing the motion $(\boldsymbol{\alpha})$ (a) and the elastic couplings $(\boldsymbol{\beta})(\mathrm{b})$
Figure 6(a) illustrates the process of optimizing the Bézier parameters $\boldsymbol{\alpha}$ implemented in MATLAB. For a given parameter set $\boldsymbol{\alpha}^{i}$ the limit cycle is determined in the hybrid zero dynamics by trapezoidal quadrature of Eq. (64) at $n_{\theta}$ sampling points and subsequent evaluation of the fixed point Eq. (69). Afterwards, the state variables of the hybrid zero dynamics $\left(\theta, L_{1}\right)$ are mapped onto the state variables and angular accelerations of the full multibody system $(\mathbf{q}, \dot{\mathbf{q}}, \ddot{\mathbf{q}})$ by using Eq. (58) - (60) and (4). The respective joint torque $\mathbf{u}$ is determined by evaluating Eq. (5). The objective function is evaluated by trapezoidal quadrature of Eq. (75) at $n_{\theta}$ sampling points with linear interpolation of zero crossings. The constraint function is also evaluated at $n_{\theta}$ sampling points. Objective and constraint function are forwarded to MATLAB's implementation of the SQP algorithm, which calculates the parameter set $\boldsymbol{\alpha}^{i+1}$ for the next iteration using a quasi Newton approach. The optimum described by the parameter set $\boldsymbol{\alpha}^{*}$ is reached, if the termination tolerance for the function TolFun and for the constraints TolCon are fulfilled. At this point it has to be mentioned, that there is no iteration in the determination of the limit cycle, neither in the full multibody system nor in the hybrid zero dynamics. The limit cycle is given directly by Eq. (69). Hence, this approach is numerically very efficient and allows for the optimization of the elastic couplings in an outer loop.

\subsection{Structure optimization}

While the Bézier parameters $\boldsymbol{\alpha}$ are changed and optimized separately for each speed $\bar{v}$, the structure and therefore the parameters of the elastic couplings $\boldsymbol{\beta}$ are fixed after design and independent of movement speed. Hence, they have to be a trade-off for different speeds. In order to find an energy efficient robot in a broad region of operation, the elastic couplings $\boldsymbol{\beta}$ are optimized for the speed range $\bar{v}_{i} \in[0.3,2.3] \mathrm{m} / \mathrm{s}$. As objective function

$f_{\min _{\boldsymbol{\beta}}}(\boldsymbol{\beta})=\bar{c}_{T}=\frac{1}{n_{\bar{v}}} \sum_{i_{\bar{v}}=1}^{n_{\bar{v}}} f_{\min _{\alpha}}\left(\boldsymbol{\alpha}^{i_{\bar{v}}}, \boldsymbol{\beta}\right)$

the mean specific cost of transport for the speed range is selected. Whereas the interval $[0.3,2.3] \mathrm{m} / \mathrm{s}$ is subdivided in $n_{\bar{v}}$ subintervals evaluated at their midpoints.

Fig. 6(b) illustrates the process of optimizing the elastic coupling parameters $\boldsymbol{\beta}$ implemented in MATLAB. For a given parameter set $\boldsymbol{\beta}^{i}$ the motion $\boldsymbol{\alpha}^{i_{\bar{v}}}$ is optimized for $n_{\bar{v}}$ single speeds $\bar{v}_{i}$ separately. The optimization of motion is performed in an inner loop displayed in Fig. 6(a). The objective function is determined by averaging the single specific costs of transport of the different speeds. Since the process of optimizing motion adds numerical noise, the optimization problem becomes non-smooth and nonlinear. It is solved by a gradient free direct search algorithm called pattern search. 
The approach varies consecutively each parameter around the reference point $\boldsymbol{\beta}^{i}$ by steps $\Delta \boldsymbol{\beta}$ of the same magnitude. The point in the mesh with the lowest value becomes the new reference point, if the new point equals the old point, the mesh size will be halved until the pattern search termination mesh size tolerance TolMesh is reached.

\section{Results}

In this section the effect of elastic couplings on the robot is presented. The focus of the investigation is on the reduction of the specific cost of transport. The robot's motion and energy turnover are analyzed to identify the major energy loss component as well as to suggest counter measures (Sec. 4.1). Subsequently the influence of the elastic couplings' topology, the legs' inertia, joint friction and the coefficients of static friction as well as static power are presented (Sec. 4.2). A detailed discussion of those parameters is provided in Appx. A. Finally, the influence of the elastic coupling on stability and sensitivity with respect to disturbances of the robot's motion is analyzed (Sec. 4.3).

\subsection{Reduction of cost of transport}

In order to isolate the effect of elastic couplings a robot without elastic couplings is considered first. Its motion $(\boldsymbol{\alpha})$ is optimized for each speed by the process described in Sec. 3.1. Fig. 7 illustrates the resulting motions for the lowest $(\bar{v}=$ $0.3 \mathrm{~m} / \mathrm{s})$, medium $(\bar{v}=1.3 \mathrm{~m} / \mathrm{s})$ and highest $(\bar{v}=2.3 \mathrm{~m} / \mathrm{s})$ considered speed at five equidistant instances of time.

The step length as well as the inclination angle of the trunk of the robot increase with increasing speed. In order to investigate the energy turnover of the robot, the specific energies $^{6}$

$$
\begin{aligned}
c_{T} & =\frac{\sum_{i=1}^{2} \int_{0}^{T} \max \left(c_{\text {stat }} u_{i}^{2}+u_{i} \dot{q}_{i}, 0\right) \mathrm{d} t}{m g r_{2_{x}}\left(\theta^{-}\right)}, \\
e_{\text {mech }}^{+} & =\frac{\sum_{i=1}^{2} \int_{0}^{T} \max \left(u_{i} \dot{q}_{i}, 0\right) \mathrm{d} t}{m g r_{2_{x}}\left(\theta^{-}\right)}, \\
e_{\text {mech }}^{-} & =-\frac{\sum_{i=1}^{2} \int_{0}^{T} \min \left(u_{i} \dot{q}_{i}, 0\right) \mathrm{d} t}{m g r_{2_{x}}\left(\theta^{-}\right)}, \\
e_{\text {imp }} & =-\frac{E_{\text {kin }}\left(\theta^{+}\right)-E_{\text {kin }}\left(\theta^{-}\right)}{m g r_{2}\left(\theta^{-}\right)}, \\
e_{\text {ela }} & =\frac{\max \left(V_{\text {ela }}\right)-\min \left(V_{\text {ela }}\right)}{m g r_{2_{x}}\left(\theta^{-}\right)}, \\
e_{\text {stat }} & =\frac{\sum_{i=1}^{2} \int_{0}^{T} c_{\text {stat }} u_{i}^{2} \mathrm{~d} t}{m g r_{2_{x}}\left(\theta^{-}\right)}
\end{aligned}
$$

\footnotetext{
6 In this paper the term specific energies refers to the quantities introduced in Eq. (82) - Eq. (87) although some of them are works (process, not state quantities).
}

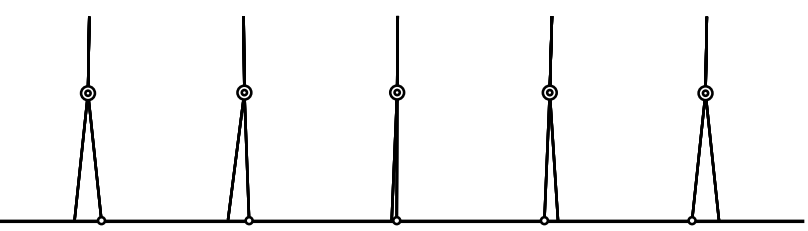

(a) $\bar{v}=0.3 \mathrm{~m} / \mathrm{s}$

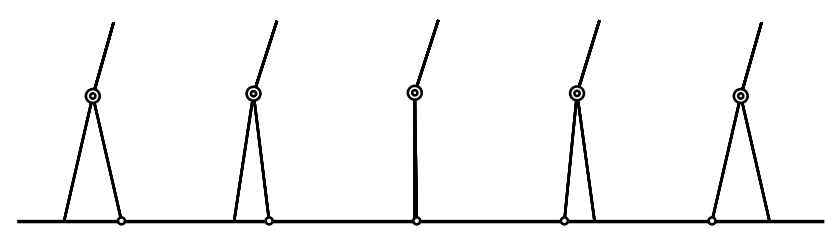

(b) $\bar{v}=1.3 \mathrm{~m} / \mathrm{s}$

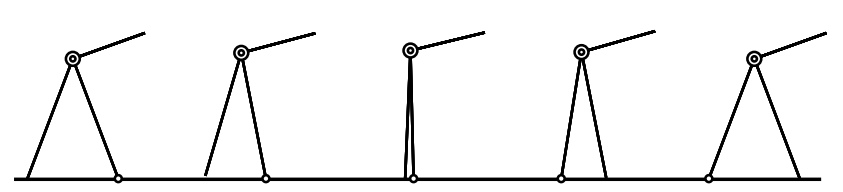

(c) $\bar{v}=2.3 \mathrm{~m} / \mathrm{s}$

Fig. 7 Comparison of the robot's motion without elastic coupling at three different speeds $\bar{v}$ at five equidistant instances of time

are introduced. Analogous to the specific cost of transport $c_{T}$, these quantities are related to the robots weight $m g$ and the distance traveled which is the step length $r_{2_{x}}\left(\theta^{-}\right)$. While the specific cost of transport $c_{T}$ corresponds to the electric energy intake of the actuators, the specific positive mechanical work $e_{m e c h}^{+}$corresponds to the mechanical energy input in motor or accelerating mode and the specific negative mechanical work $e_{m e c h}^{-}$to the mechanical energy takeout in generator or breaking mode. The specific impact loss $e_{i m p}$ is defined by the loss of kinetic energy during the impact and the specific elastic energy $e_{\text {ela }}$ by the maximum energy stored in the elastic coupling. The specific static energy $e_{\text {stat }}$ is defined by the heat loss in the resistance of the actuator's armature and corresponds to the electric energy effort necessary to statically supply a torque.

Figure 8 depicts the specific energies of the robot without elastic coupling. The specific cost of transport $c_{T}$ increases with speed $\bar{v}$ and consists mainly of specific positive mechanical work $e_{m e c h}^{+}$. At a speed of $\bar{v}=2.3 \mathrm{~m} / \mathrm{s}$ the specific positive mechanical work contributes $81.1 \%$ of the specific cost of transport. This implies that the positive mechanical work $e_{m e c h}^{+}$is not negligible and the specific static work $e_{\text {stat }}$, commonly used in optimal control approaches $[18,31]$ is not a suitable objective function, if energy efficiency is considered. The energy input by positive specific mechanical work $e_{m e c h}^{+}$is primarily dissipated by the specific impact loss $e_{\text {imp }}$. At a speed of $\bar{v}=2.3 \mathrm{~m} / \mathrm{s} 94.8 \%$ of the specific positive mechanical work is consumed by the specific impact loss. This implies that the optimization tries to minimize the braking mode of the actuators which means that a strategy to reduce the specific cost of transport has to 


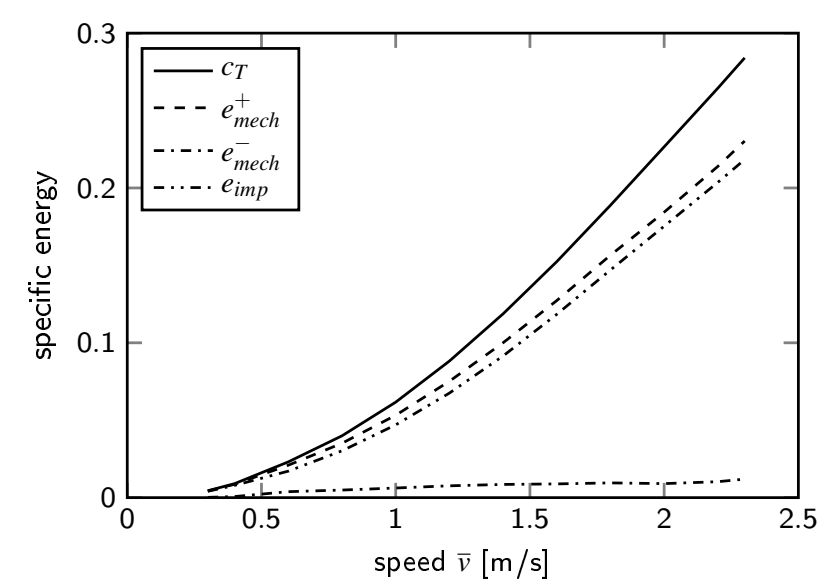

Fig. 8 Comparison of the specific energies of the robot without elastic coupling

decrease the specific positive mechanical work by reducing the specific impact loss.

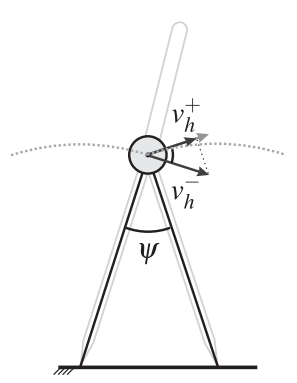

(a)

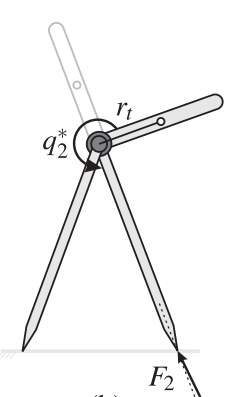

(b)

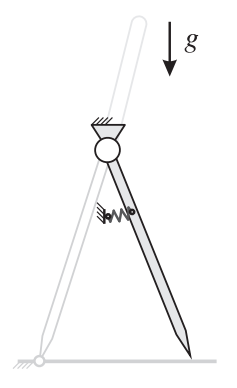

Fig. 9 Explanation of the impact loss by an Fig. 10 Explanation of inverted mathematical pendulum (a) and a the double step fremultibody model with extended trunk (b)

In order to reduce the impact loss, its origin is investigated with the simplest walking model consisting of an inverted mathematical pendulum as depicted in Fig. 9(a). During the single support phase the hip, containing the total mass, moves on a circular path around the foot of the massless stance leg. The double support phase corresponds to the transition from one circular path to the next meaning that the impact is described by a projection of the hip velocity $v_{h}^{+}=v_{h}^{-} \cos \psi$ into the new tangential direction. Therefore, the specific impact loss of the inverted pendulum

$e_{i m p_{i p}}=\frac{\left(v_{h}^{-}\right)^{2}}{2 g l_{B}} \sin \psi \cos \frac{1}{2} \psi$

can be determined analytically and is valid in the stiction domain of the leg opening angle $\psi \in\left[0,2 \arctan \left(\mu_{0}\right)\right]$.

Figure 11 displays the specific impact loss of the inverted pendulum model $e_{i m p_{i p}}$ determined with Eq. (88) and the specific impact loss of the multibody model $e_{i m p}$ determined with the impact map Eq. (27). The comparison shows

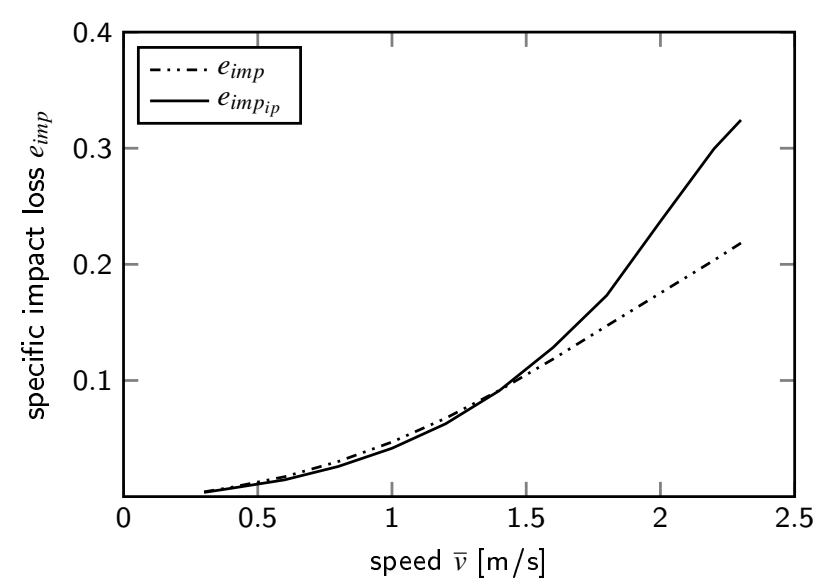

Fig. 11 Comparison of specific impact loss $e_{i m p}$ for the multibody model and the inverted pendulum model

a good match in the lower and medium speed range $\bar{v}=$ $0.3-1.6 \mathrm{~m} / \mathrm{s}$, in the higher speed range $\bar{v}=1.6-2.3 \mathrm{~m} / \mathrm{s}$ the inverted pendulum model systematically overestimates the specific impact loss. The deviation originates from the different positions of the center of mass of the inverted pendulum model and the multibody model. The inverted pendulum model assumes the center of mass of the robot in the hip. In the multibody model the trunk of the robot contributing $60 \%$ of the total mass has its center of mass at a position far away $\left(r_{t}=0.289 \mathrm{~m}\right)$ from the hip. Figure 9(b) illustrates the configuration of the robot for a speed of $\bar{v}=2.3 \mathrm{~m} / \mathrm{s}$ at the instance of impact. The direction of the resulting impact force $F_{2}$ at foot 2 almost coincides with the leg orientation (the deviation of $5.3^{\circ}$ is due to the small mass and moment of inertia of the legs). By changing the relative orientation $q_{2}$ between the impacting swing leg and the trunk, the robot can change the lever arm $r_{t} \sin q_{2}$ of the impact force with respect to the center of mass of the trunk. With increasing lever arm more translational kinetic energy is transferred into rotational kinetic energy and less energy is lost during the impact. At a speed of $\bar{v}=2.3 \mathrm{~m} / \mathrm{s}$ the lever arm almost reaches its maximum with $q_{2}^{*}=271^{\circ}$, which corresponds to a minimum in the specific impact loss. If the trunk is parallel to the impacting leg's orientation (vanishing lever arm), the specific impact loss of the multibody model is equal to the one of the inverted pendulum model. Besides reducing the impact loss at the cost of a higher static torque the trunk orientation changes the horizontal center of mass position of the robot $r_{C o M_{x}}$ and so the lever arm of weight and the applied torque for the robot's rotation about foot 1 .

Figure 12 depicts the mean center of mass position $\bar{r}_{C o M_{x}}$ of the robot. It corresponds to the energy source of the rotation about the stance leg (cf. Eq. (2)) and is necessary to overcome the impact loss $1-\delta_{0}$ (cf. Eq. (66)). Summing up, the inclination angle of the trunk results in a higher applied torque for the robot's rotation, the impact loss of the robot 


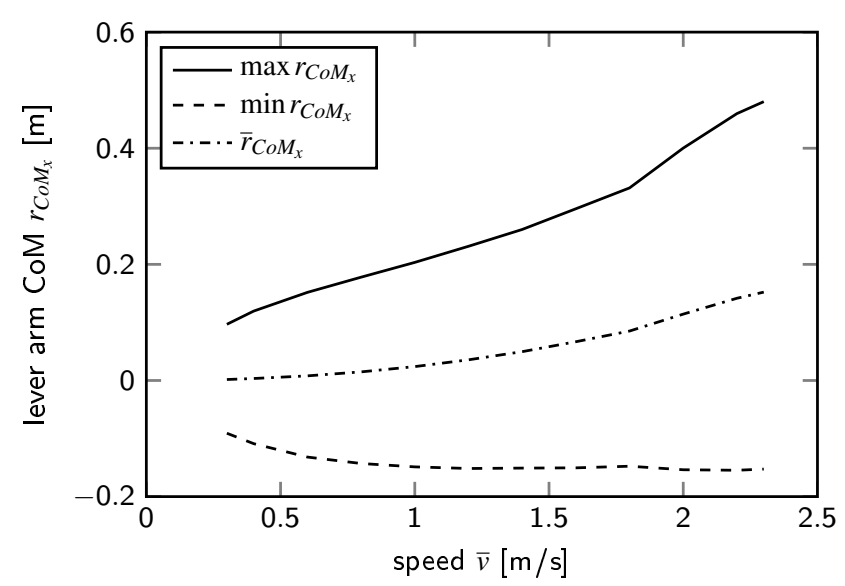

Fig. 12 Horizontal center of mass position $r_{C o M_{x}}$ as lever arm of weight and applied torque for the robot's rotation

is reduced by the orthogonal orientation of the trunk with respect to the swing leg and can be described pretty well by Eq. (88) for small trunk inclination angles.

On the other hand Eq. (88) suggests an opportunity to reduce the specific impact loss. By reducing the robot's leg opening angle $\psi$ and therewith the step length $r_{2_{x}}\left(\theta^{-}\right)$the specific impact loss of the inverted pendulum $e_{i m p_{i p}}$ decreases and finally vanishes. This raises the question why the optimization does not converge to this solution. Since the robot has to walk at a specific speed, a decrease of step length means an increase of step frequency. In order to evaluate the double step frequency ${ }^{7}$ of the optimized motion, the swing leg, which is the body with the highest acceleration in the single support phase, is modeled as physical pendulum (cf. Fig. 10). By linearizing the equation of motion about the lower rest position, the eigenfrequency

$f_{S M_{0}}=\frac{1}{2 \pi} \sqrt{\frac{g r_{l}+k_{l} l / m_{l}}{i_{l}^{2}+r_{l}^{2}}}$

of the swing leg model can be determined using the leg's radius of gyration $i_{l}$. In the entire speed range the double step frequency $f_{D S}$ of the optimized motion of the robot is significantly above the eigenfrequency $f_{S M_{0}}$ of the swing leg model (cf. Fig. 13). At a speed of $\bar{v}=1.8 \mathrm{~m} / \mathrm{s}$ the double step frequency equals 2.86 times the eigenfrequency of the swing leg model. This means the optimized motion is already far above resonance operation and to diverge further by doing smaller steps is not energetically efficient. However, the swing leg model also shows an opportunity of increasing the eigenfrequency of the swing leg model by introducing an elastic coupling of the swing leg with the stiffness $k_{l \_l}$ (cf. Eq. (89)).

In the remainder of this section a robot with linear elastic coupling between the legs is investigated (cf. Fig. 2(a)).

\footnotetext{
7 One double step corresponds to one cycle of walking and consists of one step with the right and one step with the left leg.
}

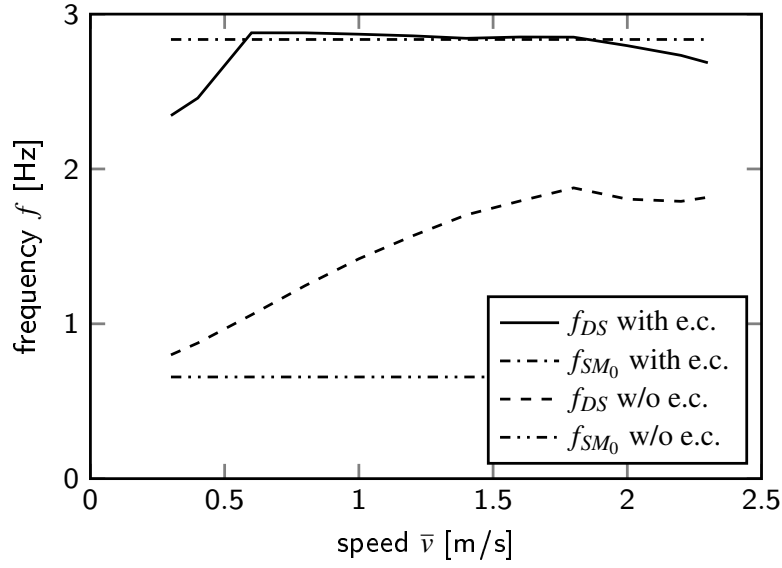

Fig. 13 Comparison of the double step frequency $f_{D S}$ of the optimized motion with the eigenfrequency $f_{S M_{0}}$ of the swing leg model for the robot with and without elastic coupling

The robot's motion $(\boldsymbol{\alpha})$ for each speed as well as the elastic coupling $(\boldsymbol{\beta})$ are optimized by the process described in Sec. 3. This results in the movement speed independent stiffness $k_{l_{-} l}=940 \mathrm{Nm} / \mathrm{rad}$ as well as the associated motions.

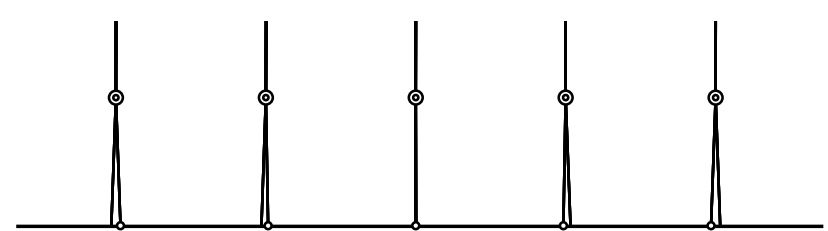

(a) $\bar{v}=0.3 \mathrm{~m} / \mathrm{s}$

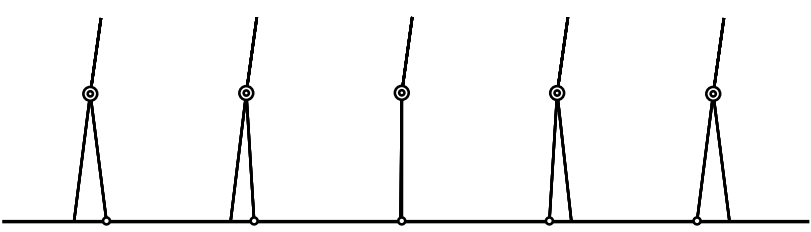

(b) $\bar{v}=1.3 \mathrm{~m} / \mathrm{s}$

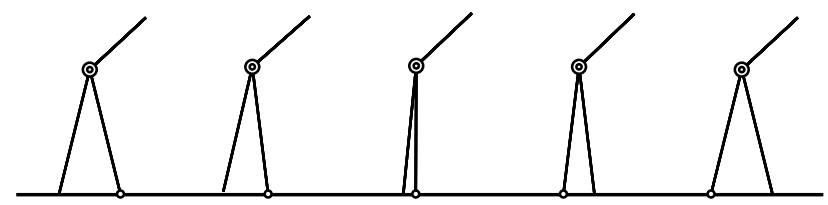

(c) $\bar{v}=2.3 \mathrm{~m} / \mathrm{s}$

Fig. 14 Comparison of the robot's motion with elastic coupling at three different speeds $\bar{v}$ at five equidistant instances of time

Figure 14 illustrates the resulting motions for the lowest $(\bar{v}=0.3 \mathrm{~m} / \mathrm{s})$, medium $(\bar{v}=1.3 \mathrm{~m} / \mathrm{s})$ and highest $(\bar{v}=$ $2.3 \mathrm{~m} / \mathrm{s})$ considered speed at five equidistant instances of time. By comparing the kinematics on position level of the motion of the robot without elastic coupling (Fig. 7) with the motion of the robot with elastic coupling (Fig. 14) two patterns emerge. 


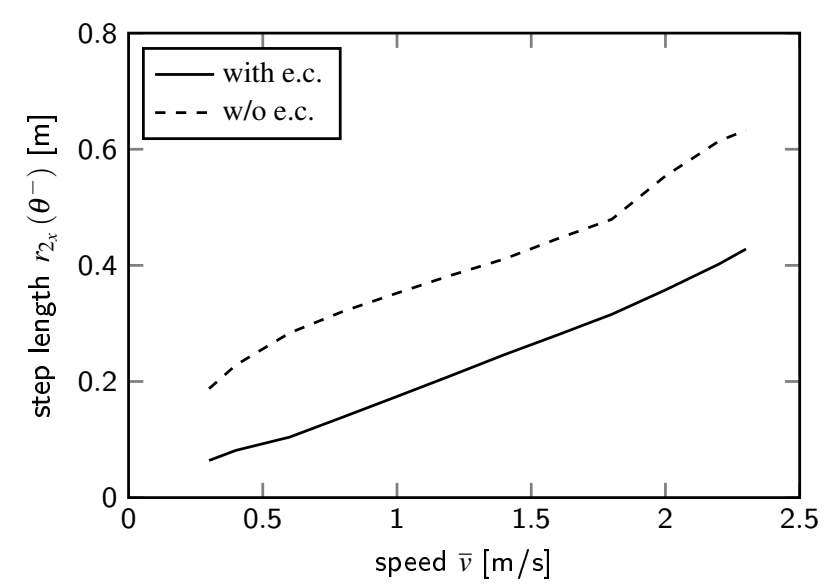

Fig. 15 Step length $r_{2_{x}}\left(\theta^{-}\right)$of the robot with and without elastic coupling

First, the step length $r_{2_{x}}\left(\theta^{-}\right)$of the robot with elastic coupling is considerably smaller than the step length of the robot without elastic coupling as depicted in Fig. 15. This indicates the expected phenomenon of reduction of impact loss. Furthermore, the step length of the robot with elastic coupling is almost a linear function of the speed, which indicates a motion with constant double step frequency.

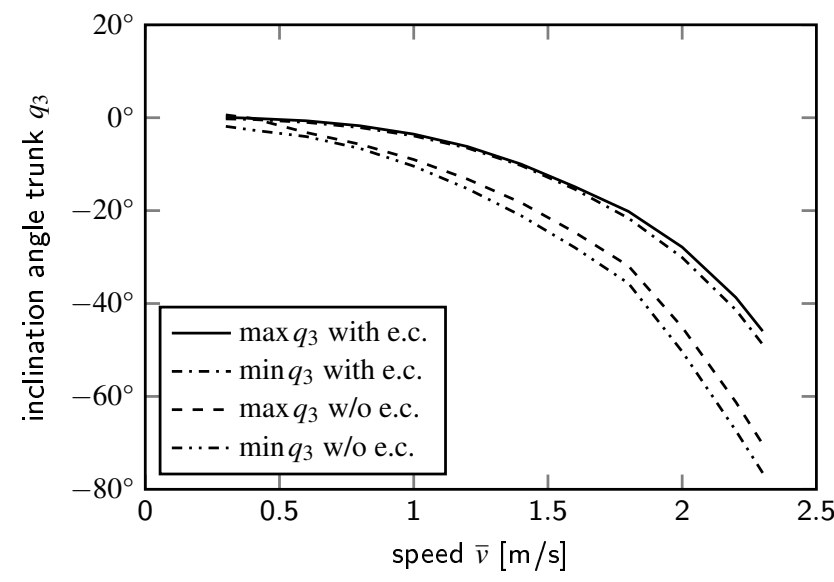

Fig. 16 Trunk inclination angle $q_{3}$ of the robot with and without elastic coupling

Second, the trunk inclination angle $q_{3}$ of the robot with elastic coupling is much smaller compared to the trunk inclination angle of the robot without elastic coupling as depicted in Fig. 16. This indicates that the impact loss is reduced elsewhere and the lever arm of the impact force does not need to be maximized. This hypothesis is further confirmed by the reduced trunk inclination angle sway; the trunk inclination angle of the robot with elastic coupling stays almost constant.

Figure 13 displays the double step frequency $f_{D S}$ of the optimized motion and the eigenfrequency $f_{S M_{0}}$ of the swing leg model of the robot with and without elastic couplings. Over a broad speed range $(\bar{v}=0.6-2.0 \mathrm{~m} / \mathrm{s})$ the double step frequency of the optimized motion is within a neighborhood of $1.5 \%$ of the eigenfrequency of the swing leg model. Therefore, we conclude that the robot moves in resonance. For higher speeds the linear model looses its eligibility and the double step frequency decreases due to nonlinear effects.

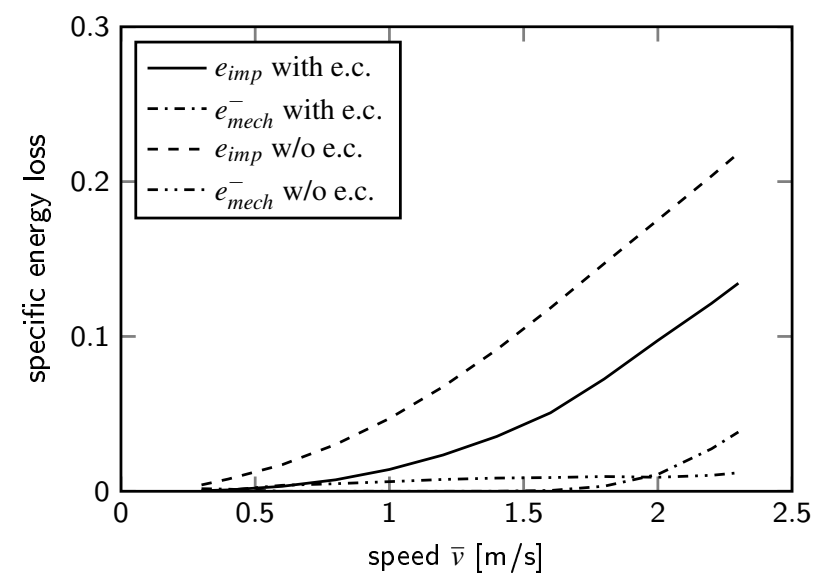

Fig. 17 Comparison of the specific energy loss of the robot with and without elastic coupling

Figure 17 shows the different specific energy losses of the robot with and without elastic coupling. The sum of the specific negative mechanical work $e_{m e c h}^{-}$and the specific impact loss $e_{i m p}$ has to be compensated by specific positive mechanical work $e_{m e c h}^{+}$and therefore determines the specific cost of transport $c_{T}$. Introducing the elastic coupling mainly reduces the specific impact loss $e_{i m p}$. This confirms the aforementioned hypothesis. Moreover, the specific negative mechanical work $e_{\text {mech }}^{-}$is reduced. In the lower and medium speed range $\bar{v}=0.3-1.6 \mathrm{~m} / \mathrm{s}$ it almost disappears and increases in the higher speed range $\bar{v}=1.6-2.3 \mathrm{~m} / \mathrm{s}$. Because of a growing step length the restoring torque of the elastic coupling and therewith the horizontal ground reaction force increase. At a speed of $\bar{v}=1.6 \mathrm{~m} / \mathrm{s}$ the ground reaction force approaches the stiction limit and therefore the robot has to break against the restoring torque. Ideally, the double step frequency $f_{D S}$ of the optimized motion should be infinitely large to avoid any impact losses. However, in this case the horizontal ground reaction force tends to infinity so that the coefficient of static friction $\mu_{0}$ restricts the reduction of the specific cost of transport by elastic couplings which is investigated in Sec. 4.2.

Figure 18 depicts the specific energy efforts of the robot with and without elastic coupling. By introduction of the elastic coupling the specific cost of transport $c_{T}$ is reduced in the entire speed range. The mean specific cost of transport $\bar{c}_{T}$ for the speed range $\bar{v}=0.3-2.3 \mathrm{~m} / \mathrm{s}$ is reduced from $\bar{c}_{T}=0.117$ to $\bar{c}_{T}=0.0509$ which is a saving of $56.6 \%$. 


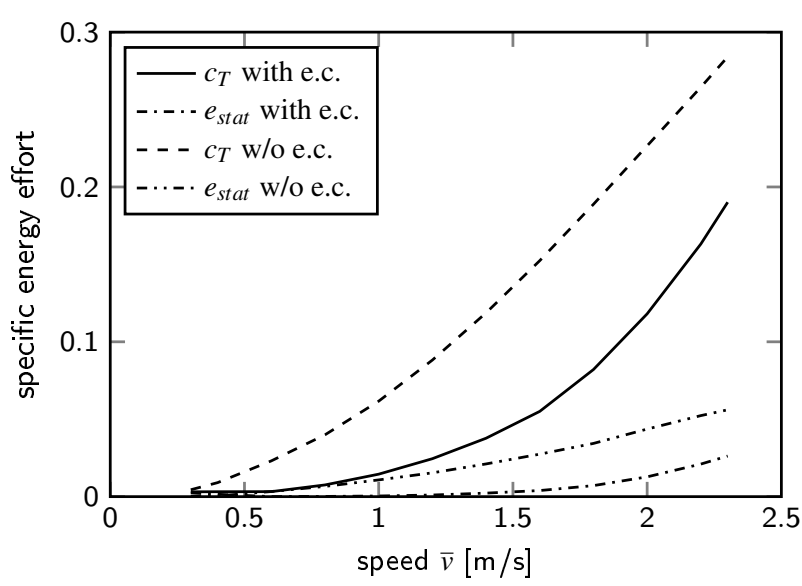

Fig. 18 Comparison of the specific energy effort of the robot with and without elastic coupling

The mean specific static energy effort $\bar{e}_{\text {stat }}$, which corresponds to the energy dissipation in form of heat in the actuator's armature resistor, is reduced from $\bar{e}_{\text {stat }}=0.0216$ to $\bar{e}_{\text {stat }}=0.00503$ which is a saving of $76.9 \%$. The elastic coupling does not only reduce the specific cost of transport significantly, it also allows for a downsizing of the actuators, which might lead to a further reduction of specific cost of transport since this changes $c_{\text {stat }}$.

Summing up, the elastic coupling increases the natural frequency of the swing leg motion and therefore allows for a resonance operation with small step length which reduces the specific impact loss and therewith the specific cost of transport significantly.

4.2 Influence of model parameters and topology of elastic couplings

A thorough study of all spring topologies as well as the model parameters has been performed. To the best of our knowledge, no systematic investigation of all possible spring topologies has been reported in literature, therefore the results are presented in detail. Furthermore, the influences of the legs' inertia, joint friction and the coefficients of static friction as well as static power are discussed. The influences of the elastic couplings' topology and the model parameters are discussed in more detail in Appx. A.

The results for all topologies from Sec. 2.1.3 are collected in Tab. 3. The minimal mean specific cost of transport $\bar{c}_{T}$ is achieved by the most complex topology, namely a combination of nonlinear elastic couplings between the legs as well as between the trunk and the legs (2nlin. $l \_l+$ $t \_l$ ). Compared to the model without elastic couplings, this topology allows for savings of $58.2 \%$. This is, however, only marginally better than a linear torsion spring between the legs (lin. $l \_l$ ), which results in savings of $56.6 \%$ and is considerably less complex to implement in a real application. Therefore this is considered as the optimal elastic coupling and used in the further parameter studies. The elastic coupling between trunk and leg cannot be used optimally in the studied speed range on its own or in combinations, because the inclination angle of the trunk is speed dependent. A detailed comparison of all topologies is presented in Appx. A.1.

The energy savings due to elastic couplings originate from an increase of the model's natural frequency. The natural frequency can be increased either by adding stiffness to the model, or by reducing the legs' inertia (cf. Eq. (89)). The question arises, if the same savings can also be achieved by changing the legs' inertia (in a practically realizable range). To study the influence of the inertia, the position of the legs' center of mass $r_{l}$ as well as their radius of gyration $i_{l}$ are varied by $\frac{2}{3}$ and $\frac{3}{2}$ times about their nominal value $\left(r_{l}^{*}, i_{l}^{*}\right)$. The influence of the position of the center of mass on the mean specific cost of transport $\bar{c}_{T}$ is significantly lower than that of the radius of gyration. The mean specific cost of transport decreases by $6.6 \%\left(i_{l}=\frac{2}{3} i_{l}^{*}\right)$ and increases by $0.8 \%$ $\left(r_{l}=\frac{2}{3} r_{l}^{*}\right), 1.2 \%\left(r_{l}=\frac{3}{2} r_{l}^{*}\right), 9.0 \%\left(i_{l}=\frac{3}{2} i_{l}^{*}\right)$ respectively. Compared to the savings by the elastic couplings, this effect is negligible. A detailed comparison is performed in Appx. A.2.

The previous studies considered an academic model without joint friction. While the increase of the model's natural frequency due to the elastic coupling results in reduced impact losses, it also accelerates the angular velocities and therewith viscous damping losses. To identify which effect predominates, the mechanical model depicted in Fig. 1 is enhanced by linear rotational joint dampers with damping coefficient $d_{J} \in[0,4,8,16] \mathrm{Nms} / \mathrm{rad}\left(d_{J}=8 \mathrm{Nms} / \mathrm{rad}\right.$ is the coefficient of the assumed reduced drive train in Sec. 2.2). This corresponds to the damping ratio $D \in$ $[0,0.158,0.316,0.632]$ of the linearized swing leg model without elastic couplings. The optimization of the motion for the model without damping results in the mean specific cost of transport of $\bar{c}_{T_{-} 0} \in[0.117,0.135,0.154,0.192]$ without the elastic coupling. The absolute saving $\Delta \bar{c}_{T} \in[0.0664,0.0622,0.0604,0.0581]$ due to the elastic coupling is almost independent of the viscous joint damping. However, the relative saving $\left(\Delta \bar{c}_{T} / \bar{c}_{T \_}\right) \in$ $[56.6 \%, 45.9 \%, 39.3 \%, 30.3 \%]$ decreases with increasing damping, since $\bar{c}_{T \_0}$ increases. For the assumed reduced drive train with joint damping coefficient $d_{J}=8 \mathrm{Nms} / \mathrm{rad}$ the mean relative saving or the reduction of the mean specific cost of transport $\bar{c}_{T}$ decreases from $56.6 \%$ to $39.3 \%$ and the reduction of the mean heat strain or the mean specific statical energy $\bar{e}_{\text {stat }}$ from $76.9 \%$ to $68.0 \%$. By the transition from the academic case without joint damping to a realistic value, the relative saving of specific cost of transport by elastic couplings decreases. However, it stays in a range relevant for 
Table 3 Comparison of different elastic couplings by parameters $\boldsymbol{\beta}$ and resulting mean specific cost of transport $\bar{c}_{T}$ with their relative saving $\Delta \bar{c}_{T} / \bar{c}_{T_{-} 0}$ related to $c_{T_{-} 0}$ the specific cost of transport of the robot without elastic coupling

\begin{tabular}{|c|c|c|c|c|c|c|c|c|c|c|}
\hline \multicolumn{2}{|c|}{ e.c. } & \multirow[t]{2}{*}{$\begin{array}{l}\text { leg }-\operatorname{leg}\left(l_{-} l\right) \\
k_{l \_l}[\mathrm{Nm} / \mathrm{rad}]\end{array}$} & \multirow[t]{2}{*}{$v_{l \_l}$} & $\begin{array}{l}\operatorname{trunk}-\operatorname{leg} \\
\varphi_{0 \_t \_l}[\mathrm{rad}]\end{array}$ & $k_{t_{-} l}^{+}[\mathrm{Nm} / \mathrm{rad}]$ & \multirow[t]{2}{*}{$k_{t_{-} l}^{-}[\mathrm{Nm} / \mathrm{rad}]$} & \multirow[t]{2}{*}{$v_{t-l}^{+}$} & \multirow[t]{2}{*}{$v_{t_{-} l}^{-}$} & $\bar{c}_{T}$ & \multirow[t]{2}{*}{$\Delta \bar{c}_{T} / \bar{c}_{T \_0}$} \\
\hline & w/o & & & & & & & & 0.1173 & \\
\hline \multirow{2}{*}{$\vec{r}$} & lin. & 940 & 1 & & & & & & 0.0509 & $56.6 \%$ \\
\hline & nlin. & 864 & 0.93 & & & & & & 0.0509 & $56.6 \%$ \\
\hline \multirow{4}{*}{ 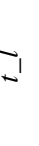 } & lin. & & & 3.42 & 201 & & 1 & & 0.1051 & $10.4 \%$ \\
\hline & nlin. & & & 3.40 & 3390 & & 7.20 & & 0.0696 & $40.7 \%$ \\
\hline & 2 lin. & & & 3.81 & 61.8 & 1820 & 1 & & 0.0769 & $34.4 \%$ \\
\hline & 2nlin. & & & 3.40 & 3590 & 3390 & 7.20 & 8.64 & 0.0691 & $41.1 \%$ \\
\hline \multirow{4}{*}{$\begin{array}{c}x^{2} \\
+ \\
+ \\
+\end{array}$} & lin. & 883 & 1 & 3.15 & 26.9 & & 1 & & 0.0506 & $56.9 \%$ \\
\hline & nlin. & 883 & 0.99 & 3.13 & 37.0 & & 1.85 & & 0.0506 & $56.9 \%$ \\
\hline & 2 lin. & 814 & 1 & 3.86 & 1.37 & 1520 & 1 & & 0.0498 & $57.5 \%$ \\
\hline & 2nlin. & 814 & 0.98 & 3.86 & 14.9 & 37800 & 11.6 & 2.31 & 0.0490 & $58.2 \%$ \\
\hline
\end{tabular}

practical application. A more detailed investigation of the effect of viscous joint damping is presented in Appx. A.3.

In Sec. 4.1 the stiction limit was identified as limiting element for the eigenfrequency $f_{S M_{0}}$ of the swing leg model, for the double step frequency $f_{D S}$ of the optimized motion and therefore for the reduction of the specific cost of transport by elastic couplings. To investigate the quantitative influence of the coefficient of static friction on the reduction of the specific cost of transport by elastic couplings, the optimization is performed for three values $\mu_{0} \in[0.6,0.8,1.0]$. While it has no influence on the motion without the elastic coupling, the savings with the elastic coupling increase from $56.6 \%$ for $\mu_{0}=0.6$ to $65.3 \%$ for $\mu_{0}=1.0$. The selected coefficient of static friction $\left(\mu_{0}=0.6\right)$ is conservatively estimated in a reasonable range and does not further increase the observed reduction in the specific cost of transport by the elastic coupling. There are reports of significant energetic improvements for models with slippage phases in recent literature [19]. This case is not investigated in the presented study. The influence of the coefficient of static friction is presented in detail in Appx. A.4.

Another parameter which influences the specific cost of transport $c_{T}$ is the coefficient of static electric power $c_{\text {stat }}$ which determines the distribution of active power into dissipated and usable mechanical power. The robot's motion changes with $c_{\text {stat }}$ since the optimization's objective function depends on it. To analyze the influence of the chosen value $c_{\text {stat }}^{*}=1.81 \mathrm{e}-3 \mathrm{~W} /(\mathrm{Nm})^{2}$, it is varied up $\left(c_{\text {stat }}=\right.$ $\left.10 c_{\text {stat }}^{*}\right)$ and down $\left(c_{\text {stat }}=0.1 c_{\text {stat }}^{*}\right)$ by one order of magnitude. Increasing $c_{\text {stat }}$ further increases the relative savings from $56.6 \%$ to $72.0 \%$, while decreasing is has only a marginal effect and changes the savings to $55.0 \%$. A detailed investigation for the coefficient of static electric power is presented in Appx. A.5.

\subsection{Stability and sensitivity}

Optimization is always a specialization. This paper focuses on energy efficiency by optimizing the robot's elastic coupling as well as the motion. This raises the question if the increase in energy efficiency sacrifices stability and increases the sensitivity with respect to disturbances of the motion. Through the use of input-output linearization, the dynamics of the robot are completely described by its hybrid zero dynamics. Hence, the stability and sensitivity of the robot's motion can be evaluated by analyzing the stability and sensitivity of the solution of the hybrid zero dynamics. The stability of the robot's motion is investigated by means of the absolute value of the Floquet multiplier of the Poincaré map of the trajectory of zero dynamics just before the instance of impact (cf. Eq. (70)). The sensitivity of the robot's motion is investigated by the relative size of the basin of attraction of the stable trajectory of the zero dynamics evaluated in the Poincaré map just before the instance of impact (cf. Eq. (71)).

Figure 19 depicts the Floquet multiplier's absolute value $\delta_{0}{ }^{2}$ for the robot with and without elastic coupling. In both cases the absolute value of the Floquet multiplier is less than one, accordingly the solution and therewith the motion of the robot is stable. Since the reduction of specific cost of transport by elastic couplings is based on the reduction of impact loss, $\delta_{0}$ has to approach the value 1 (cf. Eq. (66)). Therefore, the Floquet multiplier of the robot with elastic coupling is bigger than the version without elastic coupling. This results in a slower decay of disturbances in the total angular momentum and thus a slower transition to the desired average speed.

Figure 20 displays the relative size of the basin of attraction $s_{\mathscr{B}}$ for the robot with and without elastic coupling. For speeds above $\bar{v}=0.5 \mathrm{~m} / \mathrm{s}$ the relative size of the basin of attraction of the stable solution of the robot with elastic 


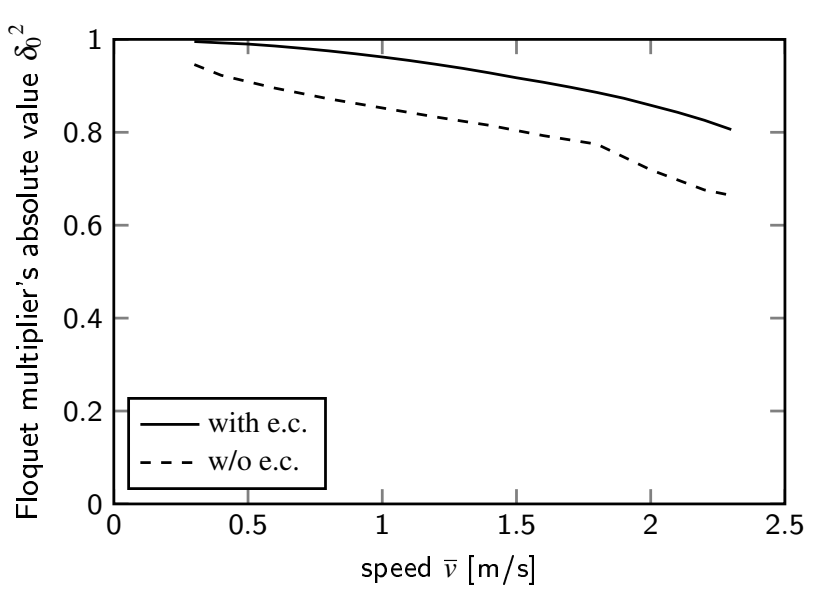

Fig. 19 Comparison of the Floquet multiplier's absolute value $\delta_{0}^{2}$ for the robot with and without elastic coupling

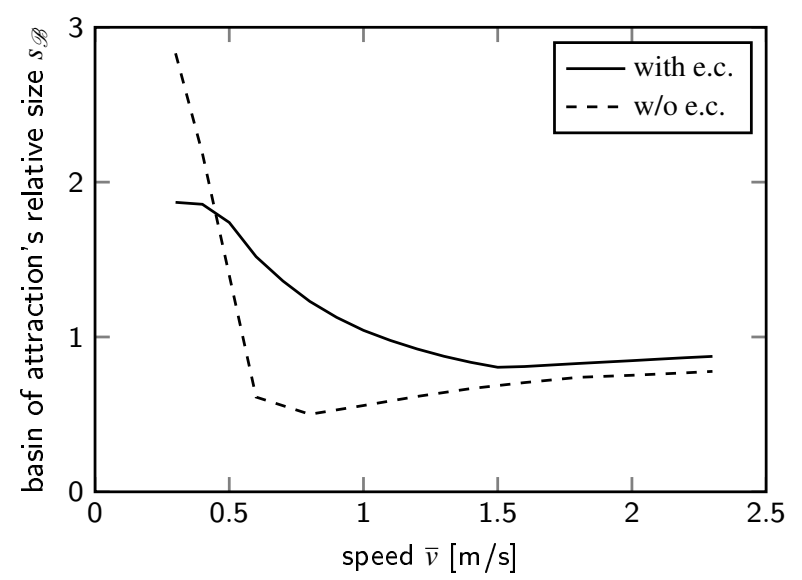

Fig. 20 Comparison of the basin of attraction's relative size $s_{\mathscr{B}}$ for the robot with and without elastic coupling

coupling is bigger compared to the version without elastic coupling. In the important medium speed range $\bar{v}=0.6-$ $1.2 \mathrm{~m} / \mathrm{s}$ the relative size of the basin of attraction of the stable solution of the robot with elastic coupling is almost twice the size of the variant without elastic coupling. The reason for this gain in size of the basin of attraction is the smaller step length which lowers the impact loss (cf. Eq. (67)) as well as the threat to fall backwards and thus raises the upper bound (cf. Eq. (72)).

The utilization of elastic couplings to reduce the specific cost of transport results in a motion with slightly slower convergence rate with respect to disturbances and even increases the size of the relative basin of attraction.

\section{Conclusion and outlook}

It is known from literature [12] that a bipedal robot will only walk efficiently if its control does not fight against its natural dynamics but allows for it to evolve. For a given robot design elastic couplings are the only possibility to shape the natural dynamics. Hence, the aim of this paper was to investigate the influence of elastic couplings on the energy efficiency of a bipedal walking robot. For this purpose an $1.80 \mathrm{~m}$ tall, $80 \mathrm{~kg}$ heavy, underactuated robot was considered, feedback controlled with input-output linearization and analyzed in the hybrid zero dynamics as proposed by [39]. The gained knowledge is transferable to every bipedal robot provided that its control allows for the evolution of natural dynamics.

In order to quantify the energy efficiency and to evaluate the influence of the elastic couplings, the specific cost of transport was introduced as energy input divided by distance traveled and weight. The energy input was defined as the integral over the electric power intake of the joint actuators. For the development of an energy efficient robot an optimization process was designed, minimizing the mean specific cost of transport in the walking speed range $\bar{v}=$ $0.3-2.3 \mathrm{~m} / \mathrm{s}$ by optimizing the motion and the elastic coupling of the robot simultaneously. The considered parameters of the elastic coupling are movement speed independent.

The energy turnover of the robot was analyzed in detail to explain the effect of reduction of specific cost of transport. For the robot without elastic couplings the energy input by the actuators is mainly dissipated by the plastic impact at touchdown of the swing leg foot in the double support phase. By an orthogonal orientation of the trunk relative to the impacting leg the impact loss is partly reduced at high speeds. By modeling the robot during the double support phase as an inverted mathematical pendulum it could be shown that the reduction of step length is a measure to further reduce the impact loss. At a given desired speed, the reduction of step length directly increases the double step frequency. By modeling the swing leg during the single support phase as a physical pendulum, it could be shown that the double step frequency of the robot without elastic coupling is far above the resonance frequency of the swing leg motion. By use of the elastic coupling between the legs the resonance frequency of the swing leg motion is increased, so that the effect of decrease of impact loss by reduction of step length can be exploited. Using the optimized elastic coupling between the legs the robot moves in resonance over a broad range of speeds and the specific cost of transport is reduced.

The mean specific cost of transport is reduced by $56.6 \%$ for the academic robot without joint damping and by $39.3 \%$ for the robot with realistic joint damping using optimal elastic coupling which is a linear torsion spring between the legs. While the relative reduction decreases with increasing joint damping, the absolute reduction is retained.

The mean static electric energy, the heat load of the actuators, is reduced by $76.9 \%$ for the academic robot without joint damping and by $68.0 \%$ for the robot with realistic joint damping using the elastic coupling between the legs. 
This allows for downsizing of the actuators and thus reducing weight and friction of the robot.

By moving the center of mass of the leg towards the foot, the relative saving of specific cost of transport is decreased insignificantly, by raising the radius of gyration and so the mass moment of inertia of the leg, the relative savings increase significantly.

The double step frequency is restricted by the stiction limit because of a suspension of the joint torques by the stance leg foot. With higher permitted coefficient of static friction in the optimization, both double step frequency and the reduction of the mean specific cost of transport increase.

Lowering the coefficient of static electric power by using a different actuator the relative saving of specific cost of transport decreases only marginally, while raising the coefficient of static power the relative saving increases notably.

The optimization of the robots motion and elastic coupling towards energy efficiency does not interfere with stability and sensitivity of the motion. Using elastic couplings the motion of the robot stays stable, however, the speed of decay of perturbations decreases as a matter of the reduced impact loss. The motion of the robot even becomes less sensitive using elastic couplings because the relative size of the basin of attraction of the stable solution increases.

It can be summarized that using elastic couplings, the specific cost of transport of the robot can be reduced significantly with slightly slower convergence rate but no loss of stability and lower sensitivity with respect to disturbances of the motion.

In the future the effect of elastic couplings on a more realistic robot model comprising of the five rigid bodies trunk, thigh and shank will be investigated. The increase of the number of rigid bodies results in an increase of number of different topologies of elastic couplings and so options to reduce the specific cost of transport. Besides the influence of elastic couplings on the walking gait their influence on the running gait will be analyzed. The ultimate aim for the robot with knees is the validation of the effect of elastic couplings in a hardware experiment.

\section{A Detailed parameter studies}

The appendix presents detailed studies of the influences of the elastic couplings' topology (A.1), the legs' inertia (A.2), viscous joint damping (A.3) and the coefficients of static friction (A.4) as well as of static electric power (A.5). The results of those studies are summarized in Sec. 4.2.

\section{A.1 Influence of the elastic coupling's topology}

In Sec. 4 the reduction of the specific cost of transport is illustrated by the example of the linear elastic coupling between the legs. In this appendix the influence of the elastic coupling's topology is investigated in detail. Therefore, all elastic couplings depicted in Fig. 2 with characteristics described by Eq. (37) are evaluated regarding their efficiency by means of the specific cost of transport $c_{T}$ and their contribution by the specific elastic energy $e_{\text {ela }}$. In each case the motion as well as the elastic coupling is optimized with the process described in Sec. 3. The elastic coupling's parameters are independent of the robot's movement speed $\bar{v}$.

The optimal nonlinear elastic coupling of the legs (nlin. $l \_l$ ) leads to a stiffness $k_{l_{-} l}=864 \mathrm{Nm} / \mathrm{rad}$ and to an exponent $v_{l_{-} l}=0.93$. In the investigated speed range this results in the same quantitative behavior as the linear elastic coupling of the legs (lin. $l \_l$ ) (cf. Tab. 3). Their difference in specific cost of transport is $0.1 \%$ and the linear configuration is easier to design. Therefore, the linear elastic coupling of the legs is used as benchmark for the remaining topologies.

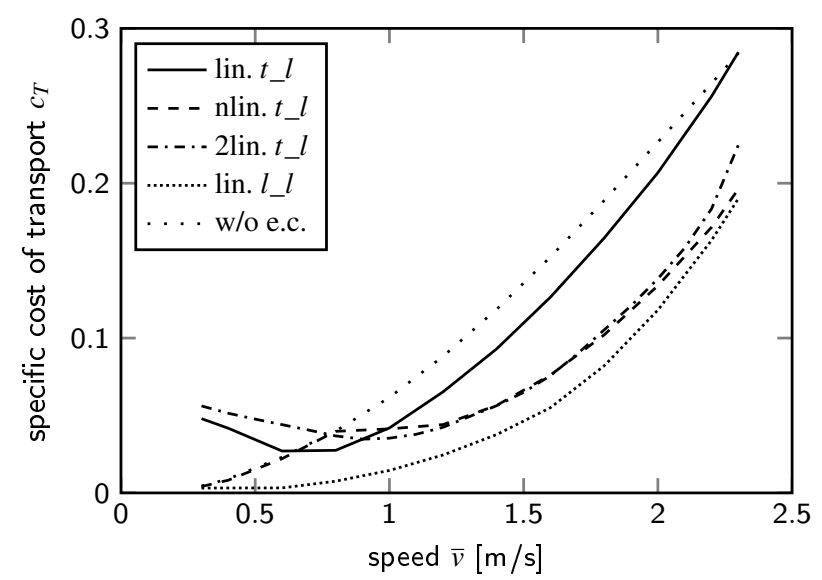

Fig. 21 Comparison of the specific cost of transport $c_{T}$ of different elastic couplings between trunk and leg $\left(t \_l\right)$

Figure 21 displays the specific cost of transport $c_{T}$ of the robot with linear (lin.), nonlinear (nlin.) and piecewise linear (2lin.) elastic couplings between trunk and leg $\left(t_{-} l\right)$ and the benchmark of the robot with linear elastic coupling between the legs (lin $l_{-} l$ ) and without elastic coupling (w/o e.c.).

The curve of the specific cost of transport of the robot with linear elastic coupling between trunk and leg (lin. $t_{-} l$ ) is for low speeds $\bar{v}=0.3-0.6 \mathrm{~m} / \mathrm{s}$ above and for medium and higher speeds $\bar{v}=0.6-$ $2.3 \mathrm{~m} / \mathrm{s}$ not far below the specific cost of transport of the robot without elastic couplings (w/o e.c.). Simultaneously, the mean specific cost of transport $\bar{c}_{T}$ is reduced compared to the robot without elastic couplings (cf. Tab. 3). Because of a movement speed dependent mean inclination angle of the trunk, the elastic coupling's movement speed independent parameters (resting angle $\varphi_{t_{-} l_{0}}=3.42 \mathrm{rad}$, stiffness $k_{t-l}=201 \mathrm{Nm} / \mathrm{rad}$ ) have to be a compromise for the regarded speed range.

The curve of the specific cost of transport of the robot with nonlinear elastic coupling between trunk and leg (nlin. $t \_l$ ) follows the curve of the specific cost of transport of the robot without elastic couplings (w/o e.c.) for low speeds $\bar{v}=0.3-0.8 \mathrm{~m} / \mathrm{s}$ and then approaches the curve of the specific cost of transport of the robot with linear elastic coupling between the legs (lin. $l_{-} l$ ). With the resting angle $\varphi_{t_{-}} l_{0}=$ $3.40 \mathrm{rad}$, the stiffness $k_{t l}=3390 \mathrm{Nm} / \mathrm{rad}$ and the exponent $v_{t l}=7.20$ the nonlinear elastic coupling between trunk and leg has the behavior of a two sided end stop. For low speeds, the amplitude of the leg swing is too low to reach the end stop. The resulting motion and specific cost of transport of the robot with nonlinear elastic coupling between trunk and leg coincide with the version without elastic couplings. For medium and higher speeds, the leg swing reaches the end stop on both sides and the specific cost of transport decreases. 
The curve of the specific cost of transport of the robot with piecewise linear elastic coupling between trunk and leg (2lin. $t \_l$ ) is above the curve of the specific cost of transport of the robot without elastic couplings (w/o e.c.) for low speeds $\bar{v}=0.3-0.8 \mathrm{~m} / \mathrm{s}$ and then almost coincides with the curve of the specific cost of transport of the robot with nonlinear elastic coupling between trunk and legs (nlin. $t_{-} l$ ). With the resting angle $\varphi_{t} l_{0}=3.81 \mathrm{rad}$ and the two stiffnesses $k_{t l}^{+}=61.8 \mathrm{Nm} / \mathrm{rad}$ and $k_{t l}^{-}=1820 \mathrm{Nm} / \mathrm{rad}$ the piecewise linear elastic coupling between trunk and leg has the behavior of an one sided end stop and mainly supports the torque of the weight of the trunk caused by its inclination angle.

The curve of the specific cost of transport of the robot with piecewise nonlinear elastic coupling between trunk and leg mainly coincides with the nonlinear variant and is omitted for the sake of clarity.

At all considered speeds $\bar{v}=0.3-2.3 \mathrm{~m} / \mathrm{s}$ the specific cost of transport of the robot with elastic couplings between trunk and leg is above the version with elastic coupling between the legs, independent of particular linear, nonlinear, piecewise linear or piecewise nonlinear characteristic of the torque angle curve. Hence, the linear elastic coupling between the legs, which was already selected as benchmark is the optimal elementary elastic coupling.

After the investigation of all single elastic couplings, the determination of the optimal combination of elastic couplings remains. For this purpose different combinations of linear, nonlinear, piecewise linear and piecewise nonlinear elastic couplings between trunk and leg and between the legs are investigated.

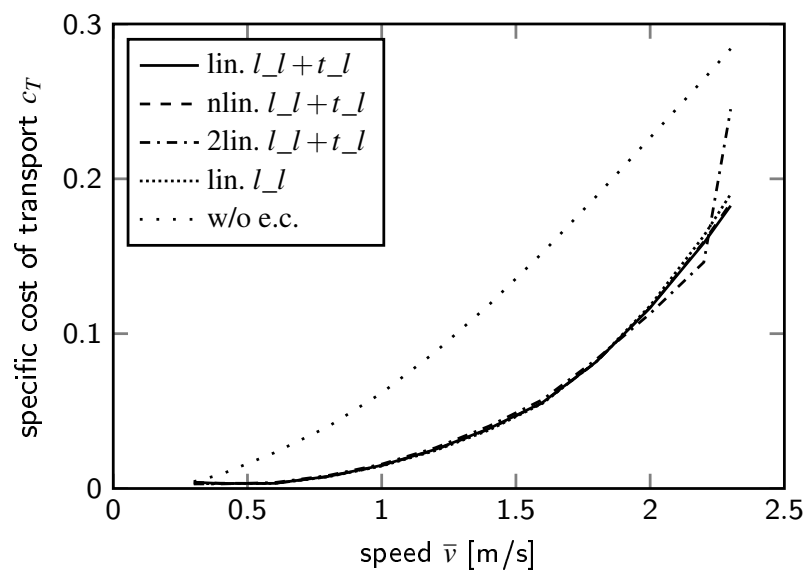

Fig. 22 Comparison of the specific cost of transport $c_{T}$ of different combinations of elastic couplings between the legs and between trunk and leg $\left(l_{-} l+t_{-} l\right)$

Figure 22 illustrates the specific cost of transport $c_{T}$ for the different combinations of elastic couplings. The mean specific cost of transport $\bar{c}_{T}$ as well as the parameters of the elastic coupling $\boldsymbol{\beta}$ for all combinations are assembled in Tab. 3 . The curves of the combinations are all very close to the curve of the robot with merely linear elastic coupling of the legs (lin. $l \_l$ ).

The curves of the combinations of linear (lin. $l \_l+t \_l$ ) and nonlinear (nlin. $l \_l+t \_l$ ) elastic couplings are slightly below the specific cost of transport of the version with linear elastic coupling of the legs (lin. $l \_l$ ) at high speeds $\bar{v}=2.0-2.3 \mathrm{~m} / \mathrm{s}$.

The curve of the combination of piecewise linear (2lin. $l \_l+t \_l$ ) elastic couplings is first below the specific cost of transport of the version with linear elastic coupling of the legs (lin. $l \_l$ ) and then increases steeply at high speeds $\bar{v}=2.0-2.3 \mathrm{~m} / \mathrm{s}$. With the resting angle $\varphi_{t_{-} l_{0}}=$ $3.86 \mathrm{rad}$ and the stiffnesses $k_{t_{-}}^{+}=1.37 \mathrm{Nm} / \mathrm{rad}$ and $k_{t_{-}}^{-}=1520 \mathrm{Nm} / \mathrm{rad}$ the component of the elastic coupling between trunk and leg has the behavior of a one sided end stop.

The curve of the combination of piecewise nonlinear (2nlin. $l \_l+$ $t_{-} l$ ) elastic couplings is equivalent to the combination of piecewise linear elastic couplings and is not displayed.

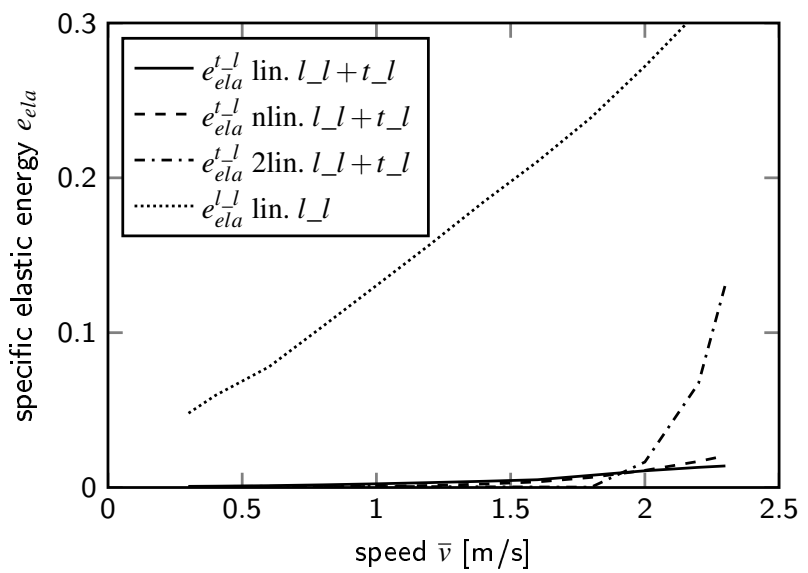

Fig. 23 Comparison of the specific elastic energy $e_{e l a}^{t} l$ stored in the elastic coupling between trunk and leg of different combinations $\left(l \_l+\right.$ $\left.t_{-} l\right)$ and the specific elastic energy $e_{\text {ela }}^{l_{l}}$ stored in the single linear elastic coupling between the legs $\left(l_{-} l\right)$

Figure 23 shows the specific elastic energy $e_{e l a}^{t}-l$ stored in the elastic coupling between trunk and leg of different combinations $\left(l \_l+t \_l\right)$ and the specific elastic energy $e_{e l a}^{l} l$ stored in the single linear elastic coupling between the legs $\left(l_{-} l\right)$. The stored elastic energy is a measure of activity of the single elastic coupling and allows for an evaluation of the contribution in the combination. The comparison of the specific elastic energy $e_{e l a}^{t} l$ stored in the elastic coupling between trunk and leg of a combination $\left(l_{-} l+t \_l\right)$ to the specific elastic energy $e_{e \overline{l a}}^{l} l$ stored in a single linear elastic coupling between the legs (lin. $l \_l$ ) shows, that the elastic coupling between trunk and leg has a minor contribution in the combinations. Merely for the piecewise linear variant the contribution rises at higher speeds, which however becomes detrimental.

The mean specific cost of transport $\bar{c}_{T}$ of the piecewise combinations (2lin. and 2nlin. $l \_l+t \_l$ ) are slightly better than the value of the single linear elastic coupling of the legs (lin. $\left.l \_l\right)(\mathrm{cf}$. Tab. 3), since the values at the edges of the speed interval $(\bar{v}=0.3 \mathrm{~m} / \mathrm{s}$ and $\bar{v}=2.3 \mathrm{~m} / \mathrm{s})$ are not used for averaging (cf. Sec. 3.2).

The elastic coupling with optimal mean specific cost of transport comprising of the piecewise nonlinear combination (2nlin. $\left.l \_l+t+l\right)$ is $1.6 \%$ better compared to the benchmark configuration comprising of the linear elastic coupling between the legs (lin. $l \_l$ ). Considering the additional effort to design and manufacture the spring between trunk and leg $(t-l)$ as well as the additional weight, it is concluded that the linear elastic coupling between the legs is still optimal for the practical application in the considered robot. By using only one elastic coupling in form of a simple mechanical spring, the mean specific cost of transport $\bar{c}_{T}$ for the speed range $\bar{v}=0.3-2.3 \mathrm{~m} / \mathrm{s}$ is reduced from $\bar{c}_{T}=0.117$ to $\bar{c}_{T}=0.0509$ which is a reduction of $56.6 \%$.

\section{A.2 Influence of the mass distribution}

In Sec. 4.1 the increase of the resonance frequency of the robot by elastic couplings was identified as a reason for the reduction of the specific cost of transport. The possible reduction of the specific cost of transport by the elastic coupling is therefore higher, the lower the resonance 
frequency of the robot without elastic coupling. In order to identify the influence of the mass distribution on the resonance frequency of the robot, it is approximated with the eigenfrequency of the swing leg motion (cf. Eq. (89)). Without elastic coupling $\left(k_{l_{-} l}=0\right)$ the resonance frequency of the robot only depends on the position of the center of mass $r_{l}$ and the radius of gyration $i_{l}$ of the leg. Hence, the investigation of the influence of the mass distribution on the reduction of the specific cost of transport by elastic coupling can be reduced to the investigation of the influence of the center of mass $r_{l}$ and the radius of gyration $i_{l}$ of the leg. Both parameters are varied by $\frac{3}{2}$ and $\frac{2}{3}$ times about their nominal value $\left(r_{l}^{*}, i_{l}^{*}\right)$.

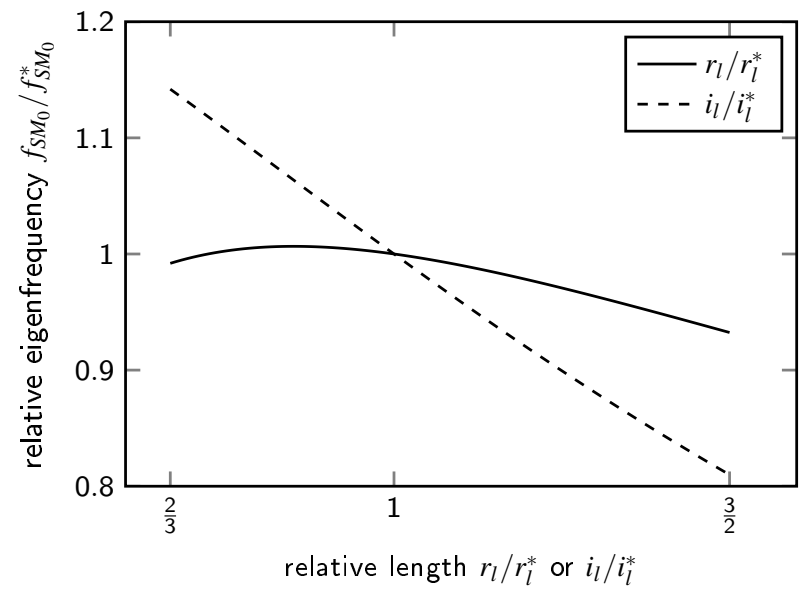

Fig. 24 Comparison of the relative eigenfrequency $f_{S M_{0}} / f_{S M_{0}}^{*}$ of the swing leg model without elastic coupling over relative center of mass $r_{l} / r_{l}^{*}$ or radius of gyration $i_{l} / i_{l}^{*}$ of the leg

Figure 24 shows the relative eigenfrequency $f_{S M_{0}} / f_{S M_{0}}^{*}$ of the swing leg model without elastic coupling over relative center of mass $r_{l} / r_{l}^{*}$ and radius of gyration $i_{l} / i_{l}^{*}$ of the leg.

In order to evaluate the influence of the position of the center of mass and radius of gyration of the leg on the saving of specific cost of transport by elastic coupling the motion as well as the elastic coupling of the robot are optimized.

The position of the center of mass $r_{l}$ of the leg has an insignificant influence on the relative saving in specific cost of transport $\Delta c_{T} / c_{T_{-} 0}$.

Figure 25 displays the relative saving in specific cost of transport $\Delta c_{T} / c_{T}$ o by elastic coupling for different radii of gyration of the leg $i_{l}$. As reference value $c_{T \_}$the specific cost of transport of the robot without elastic coupling is selected. The radius of gyration has a significant influence on the reduction of specific cost of transport by elastic couplings. Increasing the radius of gyration by $50 \%$ the reduction of the mean specific cost of transport $\bar{c}_{T}$ can be raised from $56.6 \%$ to $62.9 \%$.

\section{A.3 Influence of the viscous joint damping}

In Sec. 4 the academic case of a robot without joint friction was considered. In this section the influence of viscous joint damping on the reduction of specific cost of transport by elastic couplings is investigated. As shown in Sec. 4.1 the reduction is mainly caused by increasing the resonance frequency of the swing leg motion and thus by increasing the joint angular velocity. The joint torque caused by viscous joint damping counteracts the motion and is proportional to the angular speed. Hence, a decrease of the reduction of specific cost of transport by elastic couplings is anticipated. In order to quantify the influence of viscous joint damping the mechanical model depicted

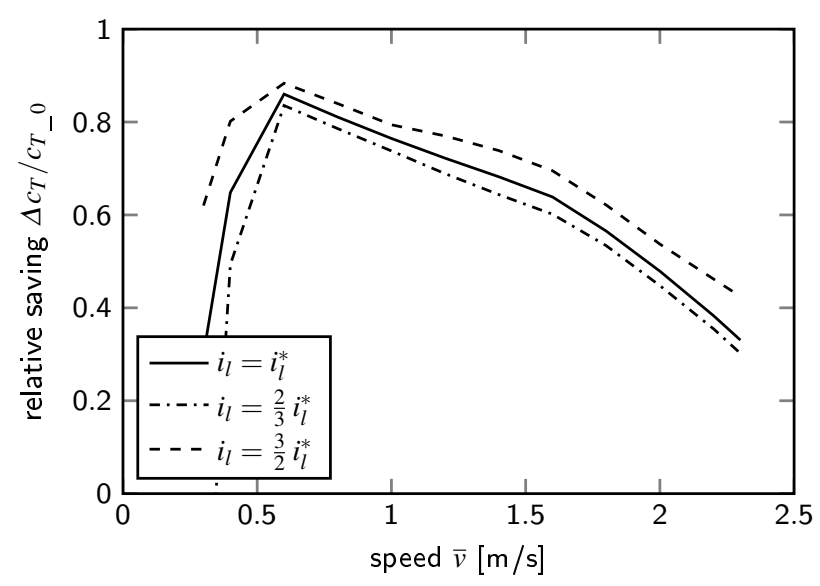

Fig. 25 Comparison of the relative saving in specific cost of transport $\Delta c_{T} / c_{T_{-} 0}$ by elastic coupling for different radii of gyration of the leg $i_{l}$

in Fig. 1 is enhanced by linear rotational joint dampers with damping coefficient $d_{J} \in[4,8,16] \mathrm{Nms} / \mathrm{rad}$ corresponding to the damping ratio $D \in[0.158,0.316,0.632]$ with respect to the eigenfrequency of the swing leg model without elastic couplings. The numerical value $d_{J}=8 \mathrm{Nm} \mathrm{s} / \mathrm{rad}$ corresponds to the value of the assumed reduced drive train (cf. Sec. 2.2). The motion as well as the elastic coupling of the robot are optimized for each joint damping coefficient if applicable.

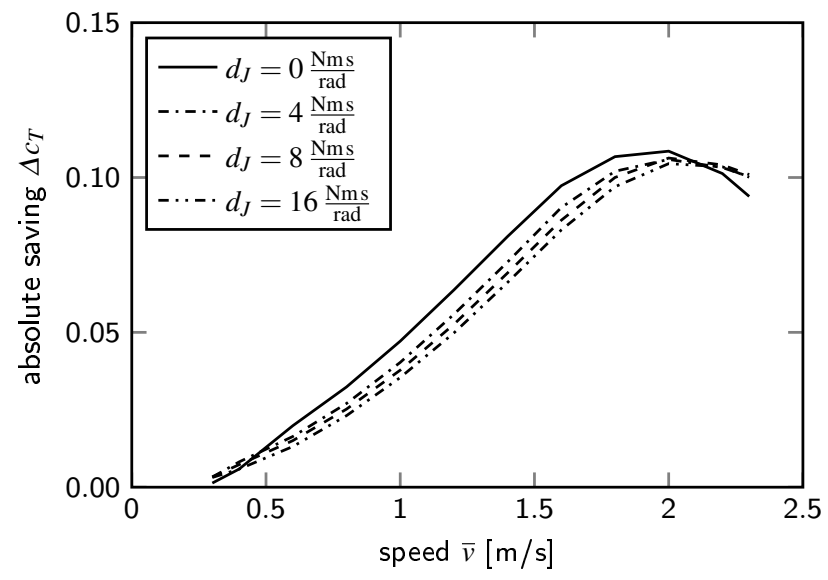

Fig. 26 Comparison of the absolute saving of specific cost of transport $\Delta c_{T}$ by elastic coupling for different joint damping coefficients $d_{J}$

Figure 26 illustrates the absolute saving of specific cost of transport $\Delta c_{T}$ by elastic coupling for different joint damping coefficients $d_{J}$. Surprisingly the absolute saving in specific cost of transport by elastic couplings depends only weakly on the joint damping coefficient.

In order to explain this circumstance, the specific energy dissipation by viscous joint damping is estimated for the joint between trunk and swing leg $\left(q_{2}\right)$ with the joint torque $T_{d}=-d_{J} \dot{q}_{2}$. The reasoning for the joint between trunk and stance leg $\left(q_{1}\right)$ follows analogously. During one step or single support phase the angle between trunk and swing leg proceeds from $q_{2}^{+}=q_{1}^{-}$just after the impact to $q_{2}^{-}$just before the the impact. At the end of the step the legs form an isosceles triangle with the edge lengths $\ell_{l}$ and $r_{2 x}^{-}=2 \ell_{l} \sin \left(\left(q_{2}^{-}-q_{2}^{+}\right) / 2\right.$ ) (cf. Fig. 1). For a small step length $r_{2_{x}}^{-}$the approximation of the circular arc with the secant and of the sine with its argument and therefore $r_{2_{x}}^{-} \approx \ell_{l}\left(q_{2}^{-}-q_{2}^{+}\right)$ 
holds. The estimation of the specific energy dissipation by viscous joint damping

$$
\begin{aligned}
c_{d} & =\frac{1}{m g r_{2 x}^{-}} \int_{q_{2}^{+}}^{q_{2}^{-}}-d_{J} \dot{q}_{2} \mathrm{~d} q_{2} \\
& \approx \frac{-d_{J}}{m g \ell_{l}} \frac{1}{q_{2}^{-}-q_{2}^{+}} \int_{q_{2}^{+}}^{q_{2}^{-}} \dot{q}_{2} \mathrm{~d} q_{2}=\frac{-d_{J}}{m g \ell_{l}} \dot{q}_{2} \approx \frac{-d_{J}}{m g} \bar{v}
\end{aligned}
$$

shows merely a dependence on the average speed $\bar{v}$ and especially not on the actual angular velocity $\dot{q}_{2}$. Therefore, the contribution of the energy dissipation by viscous joint damping to the specific cost of transport is only depending on the joint damping coefficient $d_{J}$ and equal for the robot with and without elastic couplings. This contribution consequently vanishes when the two specific costs of transport are subtracted from each other to evaluate the absolute saving of specific cost of transport by elastic coupling.

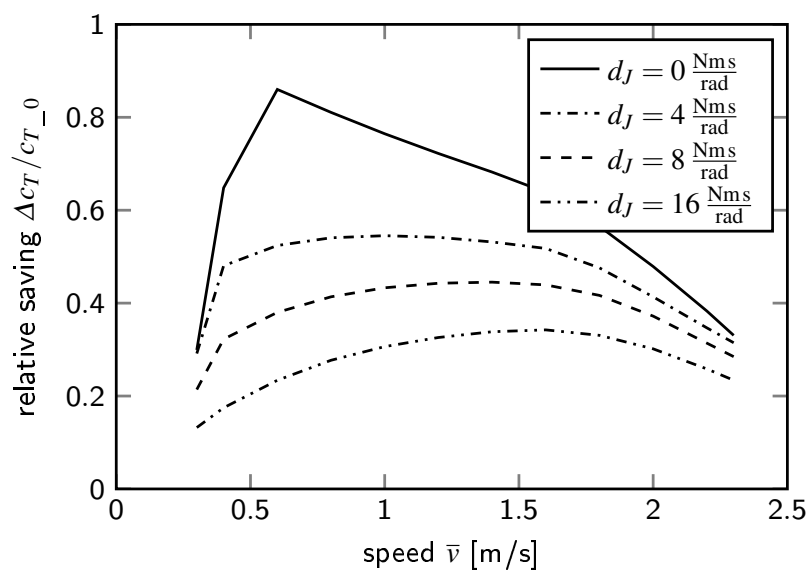

Fig. 27 Comparison of the relative saving of specific cost of transport $\Delta c_{T} / c_{T \_}$by elastic coupling for different joint damping coefficients $d_{J}$

Figure 27 shows the relative saving of specific cost of transport $\Delta c_{T} / c_{T_{-} 0}$ by elastic coupling for different joint damping coefficients $d_{J}$. Since the specific cost of transport of the robot without elastic coupling $c_{T \_}$increases with increasing joint damping coefficients $d_{J}$ while the absolute saving $\Delta c_{T}$ stays constant, the relative saving of the specific cost of transport $\Delta c_{T} / c_{T_{-} 0}$ decreases. For the assumed reduced drive train with joint damping coefficient $d_{J}=8 \mathrm{Nm} \mathrm{s} / \mathrm{rad}$ the mean relative saving or the reduction of the mean specific cost of transport $\bar{c}_{T}$ decreases from $56.6 \%$ to $39.3 \%$ and the reduction of the mean heat strain or the mean specific statical energy $\bar{e}_{\text {stat }}$ from $76.9 \%$ to $68.0 \%$. By the transition from the academic case without joint damping to a realistic value, the relative saving of specific cost of transport by elastic couplings decreases, however, it stays in a range relevant for practical application.

\section{A.4 Influence of the coefficient of static friction}

In Sec. 4.1 the stiction limit was identified as limiting element for the eigenfrequency $f_{S M_{0}}$ of the swing leg model, for the double step frequency $f_{D S}$ of the optimized motion and therefore for the reduction of the specific cost of transport by elastic couplings. In this section the quantitative influence of the coefficient of static friction on the reduction of the specific cost of transport by elastic couplings is investigated.

Figure 28 displays the specific cost of transport $c_{T}$ of the robot with and without elastic coupling for three different coefficients of

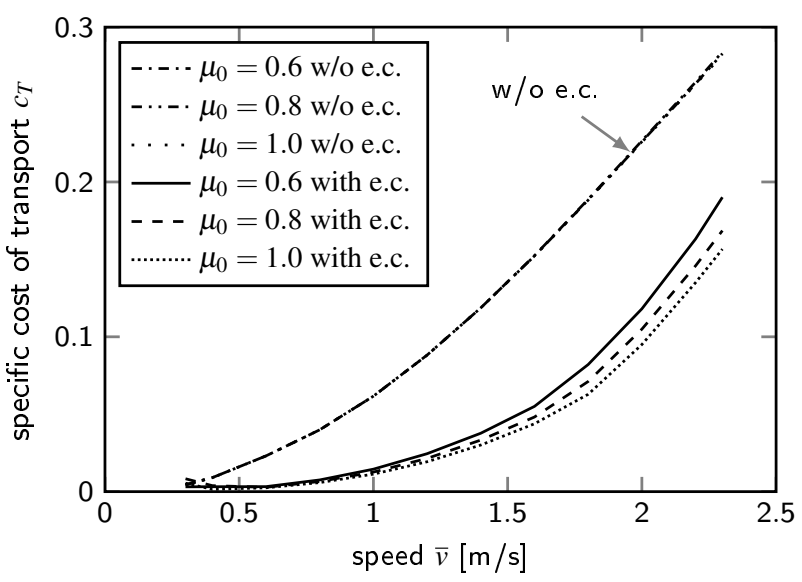

Fig. 28 Comparison of the specific cost of transport $c_{T}$ of the robot with and without elastic coupling for different coefficients of static friction $\mu_{0}$

static friction $\mu_{0} \in[0.6,0.8,1.0]$. For each parameter value the motion as well as the elastic coupling are optimized if applicable. While the particular value of the coefficient of static friction has no influence on the specific cost of transport of the robot without elastic coupling, it has an essential influence on the specific cost of transport of the robot with elastic coupling. By shifting the permitted stiction limit by the coefficient of static friction from $\mu_{0}=0.6$ to $\mu_{0}=1.0$, the mean specific cost of transport of the robot with elastic coupling is improved from $\bar{c}_{T}=0.0509$ to $\bar{c}_{T}=0.0407$ and the relative saving of mean specific cost of transport from $56.6 \%$ to $65.3 \%$. Depending on the prevailing contact conditions and safety requirements, more or less specific cost of transport can be saved. The selected coefficient of static friction $\left(\mu_{0}=0.6\right)$ is conservatively estimated, is in a reasonable range and does not further increase the observed reduction in the specific cost of transport by the elastic coupling.

\section{A.5 Influence of the coefficient of static electric power}

The coefficient of static electric power $c_{\text {stat }}$ determines the distribution of active power into dissipation power and usable mechanical power and directly affects the calculation of the specific cost of transport (cf. Eq. (44)). The used numerical value $\left(c_{\text {stat }}^{*}\right)$ is given in Tab. 1 and is derived from the actuators of the walking robot MABEL. In order to investigate the influence of the coefficient of static power its numerical value is varied up $\left(c_{\text {stat }}=10 c_{\text {stat }}^{*}\right)$ and down $\left(c_{\text {stat }}=0.1 c_{\text {stat }}^{*}\right)$ by one order of magnitude. For each parameter value the motion as well as the elastic coupling are optimized if applicable.

Figure 29 illustrates the relative saving in specific cost of transport $\Delta c_{T} / c_{T}$ o due to the elastic coupling for different coefficients of static power $c_{\text {stat }}$. As reference value $c_{T_{-} 0}$ the specific cost of transport of the robot without elastic coupling is selected. Increasing the coefficient of static electric power, for instance by selecting a stronger motor with lower transmission ratio, the relative saving of specific cost of transport increases significantly. Decreasing the coefficient of static electric power, the relative saving of specific cost of transport declines only marginally. Only in the range of high speeds $(\bar{v}=1.6-2.3 \mathrm{~m} / \mathrm{s})$ the decline is observable. The selected coefficient of static electric power $\left(c_{\text {stat }}=c_{\text {stat }}^{*}\right)$ is in a reasonable range and does especially not further increase the observed reduction in the specific cost of transport by the elastic coupling. 


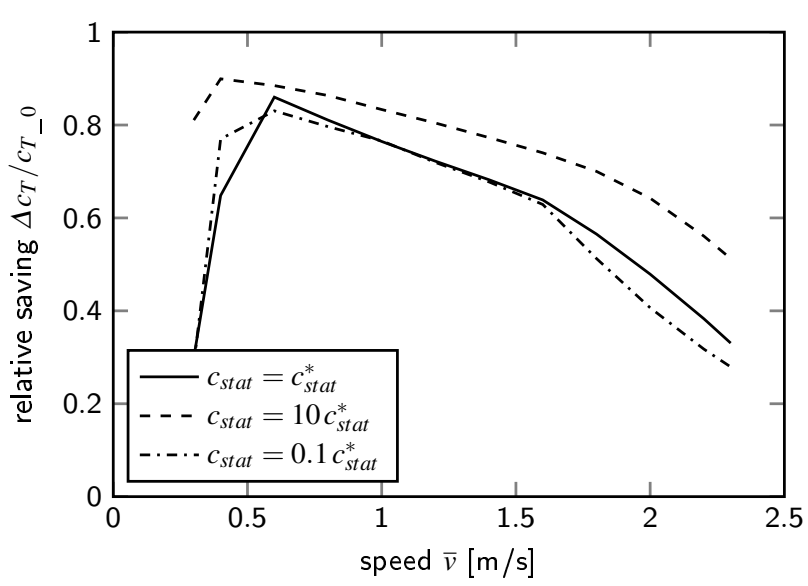

Fig. 29 Comparison of the relative saving in specific cost of transport $\Delta c_{T} / c_{T \_0}$ by elastic couplings for different coefficients of static electric power $c_{\text {stat }}$

\section{References}

1. J. Adolfsson, H. Dankowicz, and A. Nordmark. 3d passive walkers: Finding periodic gaits in the presence of discontinuities. Nonlinear Dyn., 24(2):205-229, 2001.

2. M. Ahmadi and M. Buehler. A control strategy for stable passive running. In Proc. 1995 IEEE/RSJ Int. Conf. Intell. Rob. Syst. (IROS), volume 3, pages 152-157, 1995.

3. R. M. Alexander. Three uses for springs in legged locomotion. Int. J. Rob. Res., 9(2):53-61, 1990.

4. F. Bauer. Optimierung der Energieeffizienz zweibeiniger Roboter durch elastische Kopplungen. $\mathrm{PhD}$ thesis, Karlsruhe Institute of Technolgy, 2014.

5. F. Bauer, A. Fidlin, and W. Seemann. Energy efficient bipedal robots walking in resonance. ZAMM - J. Appl. Math. Mech., 94(11):968-973, 2014.

6. F. Bauer, H. Hetzler, A. Pagel, and W. Seemann. Do Nonlinearities Enhance Stability of Bipedal Locomotion? In Proc. 2010 Int. Conf. Sim. Model. Program. Auton. Rob. (SIMPAR), pages 104-112, 2010.

7. P. A. Bhounsule, J. Cortell, A. Grewal, B. Hendriksen, J. D. Karssen, C. Paul, and A. Ruina. Low-bandwidth reflex-based control for lower power walking: $65 \mathrm{~km}$ on a single battery charge. Int. J. Rob. Res., 33(10):1305-1321, 2014.

8. E. Borzova and Y. Hurmuzlu. Passively walking five-link robot. Automatica, 40(4):621-629, 2004.

9. D. Budday, F. Bauer, and J. Seipel. Stability and Robustness of a 3D SLIP Model for Walking Using Lateral Leg Placement Control. In Proc. 2012 ASME IDETC, pages 859-866, 2012.

10. J. Calsamiglia, S. W. Kennedy, A. Chatterjee, A. Ruina, and J. T. Jenkins. Anomalous frictional behavior in collisions of thin disks. J. Appl. Mech., 66(1):146-152, 1999.

11. T. Chyou, G. F. Liddell, and M. G. Paulin. An upper-body can improve the stability and efficiency of passive dynamic walking. J. Theor. Biol., 285(1):126-135, 2011.

12. S. Collins, A. Ruina, R. Tedrake, and M. Wisse. Efficient bipedal robots based on passive-dynamic walkers. Science, 307(5712):1082-1085, 2005.

13. S. Cotton, I. M. C. Olaru, M. Bellman, T. van der Ven, J. Godowski, and J. Pratt. Fastrunner: A fast, efficient and robust bipedal robot. Concept and planar simulation. In Proc. 2012 IEEE Int. Conf. Rob. Auto. (ICRA), pages 2358-2364, 2012.

14. S. Das and A. Chatterjee. An alternative stability analysis technique for the simplest walker. Nonlinear Dyn., 28(3):273-284, 2002.
15. P. De Leva. Adjustments to Zatsiorsky-Seluyanov's segment inertia parameters. J. Biomech., 29(9):1223-1230, 1996.

16. J. C. Dean and A. D. Kuo. Elastic coupling of limb joints enables faster bipedal walking. J. Roy. Soc. Interface, 6(35):561573, 2009.

17. J. M. Donelan, R. Kram, and A. D. Kuo. Mechanical work for step-to-step transitions is a major determinant of the metabolic cost of human walking. J. Exp. Biol., 205(23):3717-3727, 2002.

18. V. Duindam and S. Stramigioli. Optimization of mass and stiffness distribution for efficient bipedal walking. In Proc. 2005 IEEE/RSJ Int. Conf. Intell. Rob. Syst. (IROS), 2005.

19. B. Gamus and Y. Or. Analysis of dynamic bipedal robot walking with stick-slip transitions. In Proc. 2013 IEEE Int. Conf. Rob. Auto. (ICRA), pages 3348-3355, 2013.

20. M. Garcia, A. Chatterjee, A. Ruina, and M. Coleman. The simplest walking model: Stability, complexity, and scaling. J. Biomech. Eng., 120(2):281-288, 1998.

21. R. Ghigliazza, R. Altendorfer, P. Holmes, and D. E. Koditschek. A simply stabilized running model. SIAM J. Appl. Dyn. Syst., 2(2):187-218, 2003.

22. M. Gomes and A. Ruina. Walking model with no energy cost. Phys. Rev. E, 83(3):032901, 2011.

23. J. W. Grizzle, J. Hurst, B. Morris, H.-W. Park, and K. Sreenath. MABEL, a new robotic bipedal walker and runner. In Proc. 2009 Am. Control Conf. (ACC), pages 2030-2036, 2009.

24. M. Hirose and K. Ogawa. Honda humanoid robots development. Philos. Trans. R. Soc. London, Ser. A, 365(1850):11-19, 2007.

25. K. Kaneko, F. Kanehiro, S. Kajita, H. Hirukawa, T. Kawasaki, M. Hirata, K. Akachi, and T. Isozumi. Humanoid robot HRP-2. In Proc. 2004 IEEE Int. Conf. Rob. Auto. (ICRA), volume 2, pages 1083-1090, April 2004.

26. A. D. Kuo. Stabilization of lateral motion in passive dynamic walking. Int. J. Rob. Res., 18(9):917-930, 1999.

27. A. D. Kuo. A simple model of bipedal walking predicts the preferred speed-step length relationship. J. Biomech. Eng., 123:264269, 2001.

28. S. Lohmeier, T. Buschmann, and H. Ulbrich. System design and control of anthropomorphic walking robot LOLA. IEEE/ASME Trans. Mechatron., 14(6):658-666, 2009.

29. T. McGeer. Passive bipedal running. Proc. R. Soc. London, Ser. B, 240(1297):107-134, 1990.

30. S. Mochon and T. A. McMahon. Ballistic walking. J. Biomech., 13(1):49-57, 1980

31. T. Narukawa, M. Takahashi, and K. Yoshida. Efficient walking with optimization for a planar biped walker with a torso by hip actuators and springs. Robotica, 29(04):641-648, 2011.

32. H.-W. Park, K. Sreenath, J. W. Hurst, and J. W. Grizzle. Identification of a bipedal robot with a compliant drivetrain. IEEE Control Syst. Mag., 31(2):63-88, 2011.

33. G. Pratt. DARPA-BAA-12-52 - Maximum Mobility and Manipulation (M3) - Actuation. DARPA. https://www.fbo.gov (2012). Accessed 23 May 2013, 2012.

34. K. Radkhah, T. Lens, and O. von Stryk. Detailed dynamics modeling of BioBiped's monoarticular and biarticular tendon-driven actuation system. In Proc. 2012 IEEE/RSJ Int. Conf. Intell. Rob. Syst. (IROS), pages 4243-4250. IEEE, 2012.

35. K. Radkhah, C. Maufroy, M. Maus, D. Scholz, A. Seyfarth, and O. von Stryk. Concept and design of the biobiped1 robot for human-like walking and running. Int. J. Humanoid Rob., 8(03):439-458, 2011.

36. M. H. Raibert. Legged robots that balance. MIT Press, 1986.

37. U. Saranlı, Ömür Arslan, M. M. Ankaralı, and Ömer Morgül. Approximate analytic solutions to non-symmetric stance trajectories of the passive Spring-Loaded Inverted Pendulum with damping. Nonlinear Dyn., 62:729-742, 2010.

38. C. M. Thompson and M. H. Raibert. Passive dynamic running. In Experimental Robotics I, pages 74-83, 1990. 
39. E. R. Westervelt, J. W. Grizzle, C. Chevallereau, J. H. Choi, and B. Morris. Feedback Control of Dynamic Bipedal Robot Locomotion. CRC Press, 2007.

40. M. Wisse, D. G. Hobbelen, and A. L. Schwab. Adding an upper body to passive dynamic walking robots by means of a bisecting hip mechanism. IEEE Trans. Rob., 23(1):112-123, 2007.

41. M. Wisse, A. L. Schwab, R. Q. van der Linde, and F. C. T. van der Helm. How to keep from falling forward: elementary swing leg action for passive dynamic walkers. IEEE Trans. Rob., 21(3):393401, 2005.

42. G. Zhong, H. Deng, and J. Li. Chattering-free variable structure controller design via fractional calculus approach and its application. Nonlinear Dyn., 81(1-2):679-694, 2015. 